\title{
A lower bound on tunnel number degeneration
}

\author{
TRENTON SCHIRMER
}

\begin{abstract}
We prove a theorem that bounds the Heegaard genus from below under special kinds of toroidal amalgamations of 3-manifolds. As a consequence, we conclude that $t\left(K_{1} \# K_{2}\right) \geq \max \left\{t\left(K_{1}\right), t\left(K_{2}\right)\right\}$ for any pair of knots $K_{1}, K_{2} \subset S^{3}$, where $t(K)$ denotes the tunnel number of $K$.
\end{abstract}

$57 \mathrm{M} 25,57 \mathrm{~N} 10$

The tunnel number $t(K)$ of a knot $K \subset S^{3}$ can be defined by the equation $t(K)+1=$ $g\left(S^{3}-\eta(K)\right)$, where $g(\cdot)$ denotes Heegaard genus and $\eta(K)$ is an open regular neighborhood of $K$. In more intuitive terms, the tunnel number of a knot is the minimal number of "tunnels" that must be drilled through $S^{3}-\eta(K)$ in order to make the resulting manifold a handlebody.

The behavior of $t(K)$ under the operation of connected sum has been studied extensively. It is not difficult to see that $t\left(K_{1} \# K_{2}\right) \leq t\left(K_{1}\right)+t\left(K_{2}\right)+1$, although it takes some work to find examples where equality is achieved in this bound; on this see Kobayashi and Rieck [5], Moriah and Rubinstein [8], and Morimoto, Sakuma and Yokota [10]. Morimoto [9] was the first to find pairs of knots in $S^{3}$ which satisfy $t\left(K_{1} \# K_{2}\right)<t\left(K_{1}\right)+t\left(K_{2}\right)$. Soon after, Kobayashi [3] constructed an infinite family of examples for which the degeneration $t\left(K_{1}\right)+t\left(K_{2}\right)-t\left(K_{1} \# K_{2}\right)$ can be arbitrarily large.

Perhaps most difficult is the task of finding lower bounds on $t\left(K_{1} \# K_{2}\right)$. Norwood [13] employed a group-theoretic argument to show that $t\left(K_{1} \# K_{2}\right) \geq 2$ for any pair of nontrivial knots in $S^{3}$, and Scharlemann and Schultens [15] subsequently used topological arguments to show $t\left(K_{1} \# \cdots \# K_{n}\right) \geq n$. In the case that $K_{1}$ and $K_{2}$ are small, Morimoto and Schultens [11] proved that $t\left(K_{1} \# K_{2}\right) \geq t\left(K_{1}\right)+t\left(K_{2}\right)$, and Kobayashi and Rieck [4] subsequently proved that this inequality holds even under the assumption that $K_{1}$ and $K_{2}$ are meridionally small. Scharlemann and Schultens [16] also proved that the lower bound $t\left(K_{1} \# K_{2}\right) /\left(t\left(K_{1}\right)+t\left(K_{2}\right)\right) \geq \frac{2}{5}$ holds for any pair of nontrivial knots in $S^{3}$ (in fact, they derive a more general analogue involving iterated connected sums there).

In this paper, we prove that

$$
t\left(K_{1} \# K_{2}\right) \geq \max \left\{t\left(K_{1}\right), t\left(K_{2}\right)\right\}
$$


for any pair of knots $K_{1}, K_{2} \subset S^{3}$. This bound was previously unknown, although there are many examples that show it to be best possible, including those of Morimoto [9], Nogueira [12], and Li and Qui [7]. Moreover, this lower bound gives a negative answer to [7, Question 1.5].

A rough outline of the strategy of our proof is as follows. Suppose without loss of generality that $\max \left\{t\left(K_{1}\right), t\left(K_{2}\right)\right\}=t\left(K_{2}\right)$ and let $K_{1} \# K_{2}$ be realized via the satellite construction with $K_{1}$ as the companion and $K_{2}$ as the pattern. This means that $K_{1} \# K_{2}$ lies in $V=\overline{\eta\left(K_{1}\right)}$, and if $h: V \rightarrow S^{3}$ is the standard unknotted embedding of the solid torus $V$, then $h\left(K_{1} \# K_{2}\right)=K_{2}$. If $\mathcal{G}$ is a thin generalized Heegaard surface of $S^{3}-\eta\left(K_{1} \# K_{2}\right)$, then $\mathcal{G}$ can be isotoped to intersect $\overline{S^{3}-V}$ in a particularly nice way. Taking into account certain information contained in the intersection $\mathcal{G} \cap\left(\overline{S^{3}-V}\right)$, we can then construct a so-called doppelgänger surface $\mathcal{Q}$ inside of a solid torus $W=\overline{S^{3}-h(V)}$ which, in certain important respects, imitates the placement of the surface $\mathcal{G} \cap\left(\overline{S^{3}-V}\right)$ in $\left(\overline{S^{3}-V}\right)$. As a result, $\mathcal{Q} \cup h(\mathcal{G} \cap V)$ forms a generalized Heegaard surface of $\overline{S^{3}-h(V)}=S^{3}-\eta\left(K_{2}\right)$ which amalgamates to a surface of lower genus than the amalgamation of $\mathcal{G}$. This yields the desired lower bound.

In Section 1, we introduce generalized compression bodies, which form the basic pieces of $\overline{S^{3}-V}-\mathcal{G}$ and $W-\mathcal{Q}$, and we prove a series of essential cutting and pasting lemmas about them. Section 2 then describes and works out the basic topology of so-called spoke graphs and spoke surfaces, which form the building blocks of the doppelgänger surface $\mathcal{Q}$. Section 3 then constructs $\mathcal{Q}$ in detail and proves that it has the desired properties, culminating in the main technical result of the paper, Theorem 3.21. In Section 4, the bound

$$
t\left(K_{1} \# K_{2}\right) \geq \max \left\{t\left(K_{1}\right), t\left(K_{2}\right)\right\}
$$

is proved (Theorem 4.1), and some topics related to it are briefly discussed.

Throughout this paper, $N(Y, X)$ denotes a closed regular neighborhood of $Y$ in $X, E(Y, X)=\overline{X-N(Y, X)}$, and $\operatorname{Fr}(Y, X)=N(Y, X) \cap E(Y, X)$, or equivalently, $\operatorname{Fr}(Y, X)=\overline{\partial N(Y)-\partial X}$. We assume throughout that $N(Y, X)$ behaves well with respect to intersection, so that $N\left(Y_{1}, X\right) \cap N\left(Y_{2}, X\right)=N\left(Y_{1} \cap Y_{2}, X\right)$. If $\mathcal{X}$ is a topological space, $|\mathcal{X}|$ denotes the number of components of $\mathcal{X}$. An embedding of manifolds $f: Y \rightarrow X$ is said to be proper if $f$ is transverse to $\partial X$ and $f(\partial Y) \subset \partial X$. A proper isotopy is a homotopy through proper embeddings (note that this does not imply that the boundary remains fixed). As an informal aid to the reader, topological spaces that are allowed to have multiple connected components will usually be denoted in calligraphic font, eg $\mathcal{A}, \mathcal{X}$, and $\mathcal{Y}$, whereas connected topological spaces will usually be denoted in standard font, eg $A, X$, and $Y$. 


\section{Generalized compression bodies}

Definition 1.1 Let $\mathcal{F}$ be a compact orientable surface, and let $\mathcal{V}=(\mathcal{F} \times I) \cup$ (2-handles) $\cup(3-$ handles $)$, where the 2 -handles are attached along essential, nonboundary parallel curves in $\mathcal{F} \times\{0\}$, and 3 -handles are attached along all spherical components of $\mathcal{F} \times I \cup(2-$ handles $)$ that are disjoint from $\mathcal{F} \times\{1\}$. Then $\mathcal{V}$ is called a generalized compression body over $\mathcal{F}$, or simply a generalized compression body. Let $\partial_{+} \mathcal{V}=\mathcal{F} \times\{1\}, \partial_{v} \mathcal{V}=(\partial \mathcal{F}) \times I$, and $\partial_{-} \mathcal{V}=\overline{\partial \mathcal{V}-\left(\partial_{+} \mathcal{V} \cup \partial_{\mathcal{V}} \mathcal{V}\right)}$. If $\mathcal{V}$ is connected and $\partial_{v} \mathcal{V}=\varnothing, \mathcal{V}$ is a compression body. If $\mathcal{V}$ is connected and $\partial_{-} \mathcal{V}=\varnothing, \mathcal{V}$ is a handlebody.

Observation 1.2 Suppose $\mathcal{V}$ is a generalized compression body, $A_{1}$ and $A_{2}$ are disjoint components of $\partial_{v} \mathcal{V}$, and $h: A_{1} \rightarrow A_{2}$ is an orientation reversing homeomorphism which preserves $\partial_{+} \mathcal{V}$. Then $\mathcal{V} / h$ is a generalized compression body over $\left(\partial_{+} \mathcal{V}\right) / h$.

Observation 1.3 If $\mathcal{V}$ is a generalized compression body and $\mathcal{W}$ is obtained by compressing $\mathcal{V}$ along a properly embedded disk $D$ such that $\partial D \subset \partial_{+} \mathcal{V}$, then $\mathcal{W}$ is again a generalized compression body. Going the other way, if $\mathcal{W}$ is obtained from $\mathcal{V}$ by attaching an oriented 1 -handle along $\partial_{+} \mathcal{V}$, then $\mathcal{W}$ is a generalized compression body.

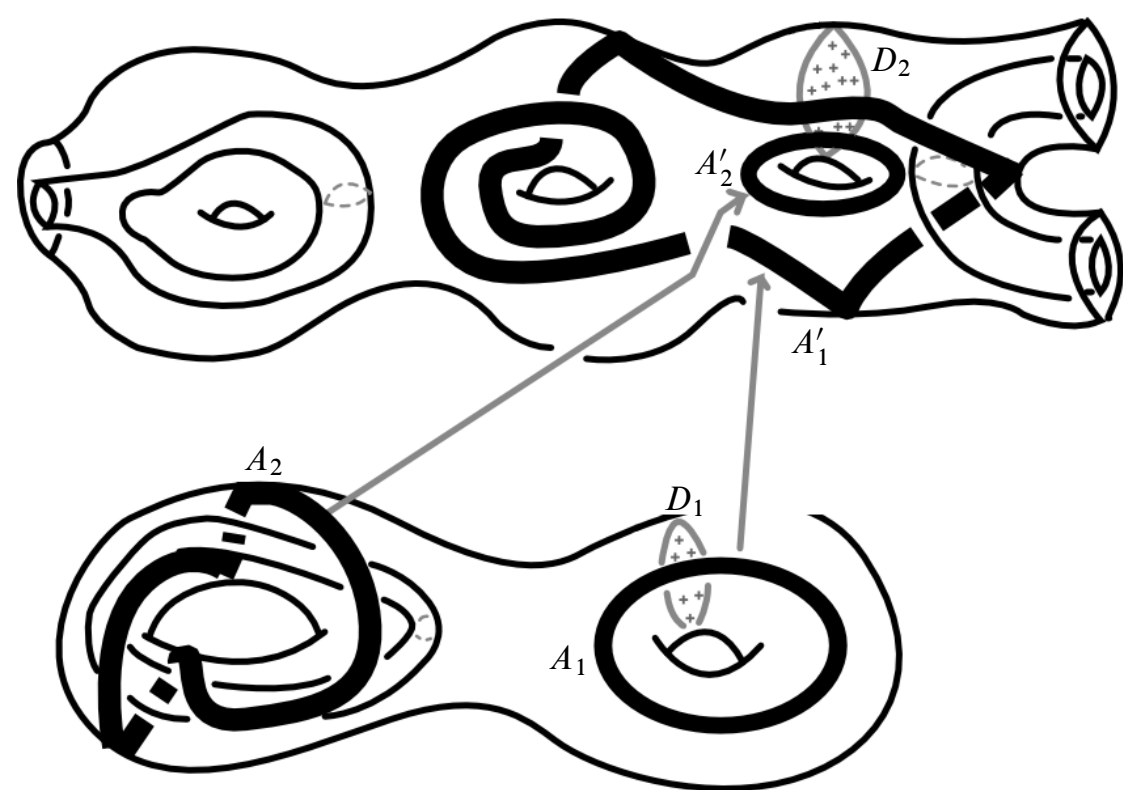

Figure 1: Primitive disk set for a paired union of annuli. 
Definition 1.4 Let $\mathcal{V}$ be a generalized compression body and let $\mathcal{A}=A_{1} \cup \cdots \cup A_{n}$ and $\mathcal{A}^{\prime}=A_{1}^{\prime} \cup \cdots \cup A_{n}^{\prime}$ be disjoint unions of annuli embedded in $\partial_{+} \mathcal{V}$ satisfying $\mathcal{A} \cap \mathcal{A}^{\prime}=\varnothing$. Let $\mathcal{D}=D_{1} \cup \cdots \cup D_{n}$ be a disjoint union of compressing disks for $\mathcal{V}$ such that $\partial \mathcal{D} \subset \partial_{+} \mathcal{V}$. If $D_{i} \cap\left(A_{i} \cup A_{i}^{\prime}\right)$ consists of a single spanning arc in one of $A_{i}$ or $A_{i}^{\prime}$ for all $1 \leq i \leq n$, and $D_{i} \cap\left(A_{j} \cup A_{j}^{\prime}\right)=\varnothing$ if $j>i$, then $\mathcal{D}$ is said to be a primitive disk set for $\mathcal{A} \cup \mathcal{A}^{\prime}$, and the component of $A_{i} \cup A_{i}^{\prime}$ which meets $D_{i}$ is said to be dual to $D_{i}$. The above orderings of the components of $\mathcal{A} \cup \mathcal{A}^{\prime}$ and $\mathcal{D}$ will be called the primitive ordering associated with $\mathcal{D}$. See Figure 1.

Remark 1.5 The choice of primitive ordering is essential to Definition 1.4, and some fixed choice is always assumed to be present when we are dealing with a primitive disk set $\mathcal{D}$ for a paired union $\mathcal{A} \cup \mathcal{A}^{\prime}$ of annuli. For the most part, however, the primitive ordering will only be specified explicitly when necessary.

Proposition 1.6 Let $\mathcal{V}$ be a generalized compression body and let $\mathcal{A}=A_{1} \cup \cdots \cup A_{n}$ and $\mathcal{A}^{\prime}=A_{1}^{\prime} \cup \cdots \cup A_{n}^{\prime}$ be disjoint unions of annuli embedded in $\partial_{+} \mathcal{V}$ satisfying $\mathcal{A} \cap \mathcal{A}^{\prime}=\varnothing$. Let $h: \mathcal{A} \rightarrow \mathcal{A}^{\prime}$ be an orientation reversing homeomorphism such that $h\left(A_{i}\right)=A_{i}^{\prime}$ for all $1 \leq i \leq n$, and suppose that $\mathcal{A} \cup \mathcal{A}^{\prime}$ admits a primitive disk set. Then $\mathcal{V} / h$ is a generalized compression body over $\left(\partial_{+} \mathcal{V}\right) / h$.

Proof We proceed by induction on $n$. In the base case $n=0$, there is nothing to prove. If $n>0$, suppose without loss of generality that $A_{n}$ is dual to $D_{n}$ (the argument is the same if $A_{n}^{\prime}$ is dual $\left.D_{n}\right)$. By Observation $1.3, E\left(D_{n}, \mathcal{V}\right)$ is again a generalized compression body, and the result of reattaching $N\left(D_{n}, \mathcal{V}\right)$ to $E\left(D_{n}, \mathcal{V}\right)$ via the map $\left.h\right|_{N\left(D_{n} \cap A_{n}, A_{n}\right)}$ again results in a generalized compression body $\mathcal{V}^{\prime}$ since this amounts to trivially attaching a ball to $\partial_{+} E\left(D_{n}, \mathcal{V}\right)$ along a disk on its boundary. But observe that $\mathcal{V} /\left.h\right|_{A_{n}}$ is obtained from $\mathcal{V}^{\prime}$ by identifying a pair of disks in $\partial_{+} \mathcal{V}^{\prime}$, which is the same as a 1 -handle attachment, so Observation 1.3 tells us that $\mathcal{V} /\left.h\right|_{A_{n}}$ is a generalized compression body. Since $D_{1} \cup \cdots \cup D_{n-1}$ was disjoint from $A_{n} \cup A_{n}^{\prime}$ and $D_{n}$, it remains a primitive disk set for $A_{1} \cup \cdots \cup A_{n-1}$ and $A_{1}^{\prime} \cup \cdots \cup A_{n-1}^{\prime}$ in $\mathcal{V} /\left.h\right|_{A_{n}}$, and the desired conclusion follows by induction.

Proposition 1.7 Suppose $\mathcal{V}$ is a generalized compression body, let $\mathcal{A}=A_{1} \cup \cdots \cup A_{n}$ be a disjoint union of annuli embedded in $\partial_{+} \mathcal{V}$, and let $\mathcal{D}=D_{1} \cup \cdots \cup D_{n}$ be a disjoint union of disks properly embedded in $\mathcal{V}$ such that $\partial \mathcal{D} \subset \partial_{+} \mathcal{V}$. If $D_{i} \cap A_{i}$ consists of a single spanning arc in $A_{i}$ for all $1 \leq i \leq n$, and $D_{i} \cap A_{j}=\varnothing$ whenever $i<j$, then manifold $\mathcal{W}$ obtained by attaching 2-handles along $\mathcal{A}$ is again a generalized compression body. 
Proof The proposition is well known in the case that $\mathcal{V}$ is a compression body. The proof in the general case here is essentially the same as that of Proposition 1.6.

Definition 1.8 An annulus $A$ properly embedded in a generalized compression body $V$ is said to be spanning if one component of $\partial A$ lies on $\partial_{+} V$, and the other lies on $\partial_{-} V . A$ is said to be horizontal if $\partial A \subset \partial_{+} V$.

Proposition 1.9 If $F$ is a compact surface and $\mathcal{A}$ is a disjoint union of incompressible spanning annuli embedded in $F \times I$, then $F \times I$ can be reparameterized so that $\mathcal{A}=\mathcal{C} \times I$ for some disjoint union of essential simple closed curves $\mathcal{C} \subset F$.

Proof This is a well known fact which often appears in the literature, so the following proof is merely a sketch. If $\mathcal{C}=\mathcal{A} \cap(F \times\{0\})$, then $\mathcal{C} \times I$ is another union of spanning annuli $\mathcal{A}^{\prime}$ such that $\mathcal{A}^{\prime} \cap(F \times\{1\})$ is isotopic to $\mathcal{A} \cap(F \times\{1\})$ in $F \times\{1\}$ (this follows from the $\pi_{1}$-injectivity of $\mathcal{A}$ and $\mathcal{A}^{\prime}$ ). This allows $\mathcal{A}^{\prime}$ to be properly isotoped so that $\partial \mathcal{A}^{\prime}$ and $\partial \mathcal{A}$ are parallel and disjoint in $\mathcal{F} \times\{0,1\}$. Since $\mathcal{A}$ and $\mathcal{A}^{\prime}$ are both incompressible, and $F \times I$ is irreducible, any simple closed curves in $\mathcal{A}^{\prime} \cap \mathcal{A}$ that are trivial in either of $\mathcal{A}^{\prime}$ or $\mathcal{A}$ can be eliminated via further isotopy of $\mathcal{A}^{\prime}$ using standard inner-most disk arguments. Again, since each component of $\mathcal{A} \cup \mathcal{A}^{\prime}$ is $\pi_{1}$-injective, any remaining components of $\mathcal{A} \cap \mathcal{A}^{\prime}$ must come from pairs of annuli $A, A^{\prime}$ with isotopic boundaries on $\mathcal{F} \times\{0,1\}$. Thus these components of $\mathcal{A} \cap \mathcal{A}^{\prime}$ can also be removed, uppermost ones first, using the fact that any incompressible horizontal annulus in $F \times I$ with parallel boundary components will cobound a solid torus with an annulus on $F \times\{1\}$. Once $\mathcal{A}^{\prime}$ has been made disjoint from $\mathcal{A}$, the components of $\mathcal{A} \cup \mathcal{A}^{\prime}$ will cobound solid tori with annuli in $F \times\{0,1\}$, so that $\mathcal{A}^{\prime}$ can finally be isotoped onto $\mathcal{A}$. Extending this proper isotopy of $\mathcal{A}^{\prime}$ to an ambient isotopy of $\mathcal{F} \times I$ yields the desired reparameterization.

Proposition 1.10 Let $\mathcal{V}$ be a compression body and let $\mathcal{A}=\mathcal{A}_{s} \cup \mathcal{A}_{h}$ be a disjoint union of incompressible annuli properly embedded in $\mathcal{V}$, so that every component of $\mathcal{A}_{s}$ is spanning and every component of $\mathcal{A}_{h}$ is horizontal. Then $E(\mathcal{A}, \mathcal{V})$ is a generalized compression body $\mathcal{V}^{\prime}$ such that $\partial_{v}\left(\mathcal{V}^{\prime}\right)=\operatorname{Fr}\left(\mathcal{A}_{s}, \mathcal{V}\right)$ and $\operatorname{Fr}\left(\mathcal{A}_{h}, \mathcal{V}\right) \subset \partial_{+} \mathcal{V}^{\prime}$. Moreover, for an appropriate ordering of the components of $\mathcal{A}_{h}=A_{1} \cup \cdots \cup A_{n}$, if we set $\operatorname{Fr}\left(A_{i}, \mathcal{V}\right)=A_{i}^{\prime} \cup A_{i}^{\prime \prime}, \mathcal{A}^{\prime}=A_{1}^{\prime} \cup \cdots \cup A_{n}^{\prime}$, and $\mathcal{A}^{\prime \prime}=A_{1}^{\prime \prime} \cup \cdots \cup A_{n}^{\prime \prime}$, then the collection $\mathcal{A}^{\prime} \cup \mathcal{A}^{\prime \prime}$ admits a primitive disk set in $\mathcal{V}^{\prime}$.

Proof From Schultens [18, Lemma 2], we have that $\mathcal{W}=E\left(\mathcal{A}_{h}, \mathcal{V}\right)$ is a union of compression bodies. The annuli $\mathcal{A}_{s}$ remain spanning in $\mathcal{W}$, and thus are disjoint from some union of disks $\mathcal{E}$ properly embedded in $\mathcal{W}$ such that $E(\mathcal{E}, \mathcal{W}) \cong \partial_{-} \mathcal{W} \times I$; 
see, eg Saito, Scharlemann and Schultens [14, Lemma 3.1.5]. By Proposition 1.9, we may assume that $E(\mathcal{E}, \mathcal{W})$ has been parameterized so that $\mathcal{A}_{s}$ has the form $\mathcal{C} \times I$ in $\partial_{-} \mathcal{W} \times I$, where $\mathcal{C} \subset \partial_{-} \mathcal{W}$ is a disjoint union of simple closed curves. Thus $\mathcal{V}^{\prime}=E\left(\mathcal{A}_{s}, \mathcal{W}\right)=E(\mathcal{A}, \mathcal{V})$ is a generalized compression body, since it is just $\partial_{-} W$ cut along $\mathcal{C} \times I \cup\{2-$ handles $\} \cup\{3-$ handles $\}$, and it satisfies $\partial_{v} \mathcal{V}^{\prime}=\operatorname{Fr}\left(\mathcal{A}_{s}, \mathcal{V}\right)$ as claimed.

We prove the second part by induction on $n$. There is nothing to prove if $n=0$. If $n>0$, then from Bonahon and Otal [2, Lemma 9], we have that $\mathcal{A}_{h}$ is boundary-compressible in $\mathcal{V}$ via some disk $D_{1}$. We may choose $D_{1}$ so that it is disjoint from $\mathcal{A}_{s}$, and we assign the label $A_{1}$ to the component of $\mathcal{A}_{h}$ which has been boundary-compressed by $D_{1}$. By the first part of this lemma, $E\left(\mathcal{A}_{S} \cup A_{1}, \mathcal{V}\right)$ is a generalized compression body, and so by induction, $\operatorname{Fr}\left(\mathcal{A}_{h}-A_{1}, \mathcal{V}\right)$ admits a primitive disk set $\mathcal{D}^{\prime}$ in $\mathcal{V}^{\prime}=E(\mathcal{A}, \mathcal{V})$ with respect to an appropriate choice of numbering for $\mathcal{A}_{h}-A_{1}$, with $A_{2}$ as the lowest indexed annulus. We may also choose (appropriately indexed) $\mathcal{D}^{\prime}$ so that $\mathcal{D}^{\prime} \cap D_{1}=\varnothing$, and since $D_{1} \cap\left(\mathcal{A}_{h}-A_{1}\right)=\varnothing$, it follows that $\mathcal{D}=\mathcal{D}^{\prime} \cup\left(D_{1} \cap \mathcal{V}^{\prime}\right)$ is a primitive disk set for $\operatorname{Fr}\left(\mathcal{A}_{h}, \mathcal{V}\right)$ in $\mathcal{V}^{\prime}$ as required.

Definition 1.11 A graph $\mathcal{X}$ embedded in a 3-manifold $M$ is said to be properly embedded if $\mathcal{X}$ is transverse to $\partial M$ and $\mathcal{X} \cap \partial M$ is a union of elements from the set of univalent vertices of $\mathcal{X}$. Also, $\mathcal{X}$ is said to be unknotted if it can be isotoped into $\partial M$ via an isotopy $\Phi: \mathcal{X} \times I \rightarrow M$ such that the function $\left.\Phi\right|_{\{t\} \times X}$ is a proper embedding for all $0 \leq t<1$.

Observation 1.12 If $X$ is an unknotted tree properly embedded in the 3-ball $B$, and $F$ is the surface which results from removing an open collar of $\partial(\overline{\partial B-N(X)})$ from $\overline{\partial B-N(X)}$, then there is homeomorphism $h: F \times I \cong E(X, B)$ such that $h(F \times\{1\})=F$ and $h(F \times\{0\})=\operatorname{Fr}(X, B)$.

Definition 1.13 Let $X$ be a graph embedded in a handlebody $V$ such that $E(X, V) \cong$ $\partial V \times I$. Then $X$ is called a spine of $V$.

Proposition 1.14 Suppose $X$ is a graph embedded in the interior of a handlebody $V$, and that there is a disjoint union of compressing disks $\mathcal{D}$ properly embedded in $V$ so that the following are true:

(1) $E(\mathcal{D}, V)$ is a union of balls.

(2) $X \cap B$ is a properly embedded, unknotted tree for each component $B$ of $E(\mathcal{D}, V)$.

(3) For each component $D$ of $\mathcal{D}, D \cap X$ is a single point on an edge of $X$.

Then $X$ is a spine of $V$. 
Proof For each component $B$ of $E(\mathcal{D}, V)$, let $\mathcal{E} \subset \partial B$ be the set of "scars" left behind by cutting along $\mathcal{D}$, ie $\mathcal{E}=N(\mathcal{D}, V) \cap B$, and let $X^{\prime}=X \cap B$. Then hypotheses 2 and 3 give us a homeomorphism $h: F \times I \rightarrow E\left(X^{\prime}, B\right)$ as per Observation 1.12, where we may take $F=\overline{\partial B-\mathcal{E}}$. These homeomorphisms can then be pasted together to form a homeomorphism between $E(X, V)$ and $\partial V \times I$.

Proposition 1.15 Suppose $V$ is a handlebody with spine $X$, and that $Y$ is a subgraph of $X$ without simply connected components. Then $E(Y, V)$ is a compression body.

Proof $E(Y, V)$ is obtained from $E(X, V) \cong \partial V \times I$ by attaching 2-handles and 3-handles along $\partial V \times\{0\}$.

Definition 1.16 A Heegaard splitting $(V, W, G)$ of a compact, connected, orientable 3-manifold $M$ is a decomposition $M=V \cup W$, where each of $V$ and $W$ is a compression body, and $G=\partial_{+} V=\partial_{+} W=V \cap W$. Also, $G$ is called a Heegaard surface. The Heegaard genus $g(M)$ of a manifold is the minimal genus of a Heegaard surface for $M$.

Definition 1.17 A generalized Heegaard splitting $\left(\left(V_{1}, W_{1}, G_{1}\right), \ldots,\left(V_{n}, W_{n}, G_{n}\right)\right)$ of a compact, orientable, connected 3-manifold $M$ is a decomposition $M=M_{1} \cup$ $\cdots \cup M_{n}$ such that the following conditions hold:

- $\left(V_{i}, W_{i}, G_{i}\right)$ forms a Heegaard splitting for the submanifold $M_{i}$.

- For all $i<j, M_{i}$ meets $M_{j}$ only along components of $\partial_{-} W_{i}$ that are identified with components of $\partial_{-} V_{j}$.

We include the case $n=1$ corresponding to standard Heegaard splittings. Let $\mathcal{G}_{+}=$ $G_{1} \cup \cdots \cup G_{n}$, and let $\mathcal{G}_{-}$denote the union of surfaces $M_{i} \cap M_{j}$ for $i<j$. Then $\mathcal{G}=\mathcal{G}_{+} \cup \mathcal{G}_{-}$is called a generalized Heegaard surface with thick surfaces $\mathcal{G}_{+}$and thin surfaces $\mathcal{G}_{-}$.

Definition 1.18 A Heegaard splitting $(V, W, G)$ is said to be:

- Stabilized if there exist compressing disks $D \subset V$ and $D^{\prime} \subset W$ such that $D \cap D^{\prime}$ is a single point.

- Reducible if there exist compressing disks $D \subset V$ and $D^{\prime} \subset W$ such that $\partial D=\partial D^{\prime}$.

- Weakly reducible if it is not stabilized or reducible, and there are compressing disks $D \subset V$ and $D^{\prime} \subset W$ such that $D \cap D^{\prime}=\varnothing$.

- Strongly irreducible if it is not stabilized and, for all compressing disks $D \subset V$ and $D^{\prime} \subset W, D \cap D^{\prime} \neq \varnothing$. 
Remark 1.19 There is a process of untelescoping a weakly reducible Heegaard splitting $(V, W, G)$ whereby it is changed into a generalized Heegaard splitting of the form $\left(\left(V_{1}, W_{1}, G_{1}\right), \ldots,\left(V_{n}, W_{n}, G_{n}\right)\right)$ satisfying $g(G)=\Sigma g\left(G_{i}\right)-\Sigma g\left(F_{i}\right)$. Conversely, given a generalized Heegaard splitting $\left(\left(V_{1}, W_{1}, G_{1}\right), \ldots,\left(V_{n}, W_{n}, G_{n}\right)\right)$ for $M$, one can always use the process of amalgamation to change it into a standard Heegaard splitting $(V, W, G)$ of $M$ satisfying the same equation. The interested reader is referred to Saito, Scharlemann and Schultens [14] for the details of these processes and a proof of the following lemma.

Proposition 1.20 (Scharlemann and Thompson [17]) Suppose that we have a weakly reducible Heegaard splitting ( $V, W, G)$ of a compact, orientable, connected, irreducible 3-manifold $M$. Then $(V, W, G)$ can be untelescoped to a generalized Heegaard splitting $\left(\left(V_{1}, W_{1}, G_{1}\right), \ldots,\left(V_{n}, W_{n}, G_{n}\right)\right)$ such that $\left(V_{i}, W_{i}, G_{i}\right)$ is a strongly irreducible splitting of $M_{i}$ for each $1 \leq i \leq n$, and the thin surfaces $F_{i}$ are incompressible in $M$ for each $1 \leq i<n$. In this case, the generalized splitting is said to be fully untelescoped. A standard Heegaard splitting that is strongly irreducible will also be considered fully untelescoped.

Proposition 1.21 (Scharlemann and Schultens [15]) If $\mathcal{G}$ is the union of the thick and thin surfaces of a fully untelescoped Heegaard splitting of $M$, and $T$ is an incompressible surface properly embedded in $M$, then $\mathcal{G}$ can be isotoped so that it meets $T$ only in simple closed curves that are nontrivial in both $\mathcal{G}$ and $T$.

\section{Spoke surfaces in the solid torus}

Convention 2.1 Throughout this section, we set $W=S^{1} \times D^{2}$ and parameterize it using polar coordinates $(\phi, r, \theta)$ with $0 \leq \phi, \theta \leq 2 \pi$ and $0 \leq r \leq 1$.

Definition 2.2 Let $X \subset W$ be an embedded graph with one central vertex $x_{0}$ at $\left(\phi_{0}, 0,0\right)$, a finite number of outer vertices $\left\{x_{1}, \ldots, x_{n}\right\} \subset\left\{\phi_{0}\right\} \times \partial D^{2}$, one radial edge connecting each outer vertex $x_{i}$ to the central vertex $x_{0}$, and one longitudinal edge $l_{i}=S^{1} \times\left\{x_{i}\right\}$ connecting $x_{i}$ to itself for $1 \leq i \leq n$. Then $X$ is said to be a connected spoke graph in $W$. A finite, disjoint union of connected spoke graphs $\mathcal{X}$ in $W$ is simply called a spoke graph, $\operatorname{Fr}(\mathcal{X}, W)$ is called a spoke surface, and $E(\mathcal{X}, W)$ is a spoke chamber.

Definition 2.3 Suppose $X$ is a connected spoke graph with central vertex at $\left(\phi_{0}, 0,0\right)$. Let $\mathcal{D}$ be a disjoint union of disks embedded in $\stackrel{\circ}{W}$ such that for each component $D$ of $\mathcal{D}$, 
- $D \cap X$ is a connected subarc of a radial edge of $X$, and

- $D \cap\left(\left\{\phi_{0}\right\} \times D^{2}\right)=D \cap X$.

Then $X \cup \partial \mathcal{D}$ is called a stabilized spoke graph with stabilizing disk set $\mathcal{D}, \operatorname{Fr}(X \cup \partial \mathcal{D})$ is a stabilized spoke surface, and $E(X \cup \partial D, W)$ is a stabilized spoke chamber. Moreover, $\overline{\partial \mathcal{D}-X}$ is called the set of stabilizing arcs of $X$.

Definition 2.4 Let $X$ be a connected stabilized spoke graph with stabilizing disk set $\mathcal{D}$ and central vertex at $\left(\phi_{0}, 0,0\right)$. Let $\mathcal{A}=E(X \cap \partial W, \partial W)$, and let $\mathcal{E}=\left(\left\{\phi_{0}\right\} \times D^{2}\right) \cap V$, where $V=E(X, W)$. Then the standard disk set of $X$ is the union of disks $\mathcal{D}_{X}=$ $\mathcal{E} \cup(\mathcal{D} \cap V) \cup \operatorname{Fr}(\mathcal{E} \cup \mathcal{A}, V)$.

Observation 2.5 The standard disk set of $X$ cuts $E(X, W)$ into a union of balls. Thus $E(X, W)$ is a handlebody.

Definition 2.6 Let $X$ be a connected, stabilized spoke graph with stabilizing disk set $\mathcal{D}$, and whose central vertex has $\phi$-coordinate $\phi_{1}$. Let $X^{\prime}$ be another connected, stabilized spoke graph disjoint from $X$ with stabilizing disk set $\mathcal{D}^{\prime}$, whose central vertex has $\phi$-coordinate $\phi_{2} \neq \phi_{1}$, and suppose the following properties are also satisfied:

- The set of longitudinal edges of $X^{\prime}$ is precisely the set of core curves of the annuli $E(X \cap \partial W, \partial W)$.

- $\mathcal{D} \cap\left(\left\{\phi_{2}\right\} \times D^{2}\right)=\varnothing=\mathcal{D}^{\prime} \cap\left(\left\{\phi_{1}\right\} \times D^{2}\right)$.

- Every component $D$ of $\mathcal{D}$ meets precisely one component $D^{\prime}$ of $\mathcal{D}^{\prime}$ in a single arc that has one endpoint on $\partial D-X$ and the other on $\partial D^{\prime}-X^{\prime}$, and conversely, each component of $\mathcal{D}^{\prime}$ meets precisely one component of $\mathcal{D}$ in this way.

- Let $h$ be the projection $S^{1} \times D^{2} \rightarrow S^{1}$. Then $\left.h\right|_{\mathcal{D}}$ is a circle-valued Morse function without singularities, and for every stabilizing $\operatorname{arc} \alpha$ of $X,\left.h\right|_{\alpha}$ is Morse with only one critical point occurring at $\alpha \cap \mathcal{D}^{\prime}$. The corresponding condition holds for the stabilizing disks and arcs of $X^{\prime}$.

Then $X$ and $X^{\prime}$ are said to be dual to one another. See Figure 2.

Remark 2.7 One way to obtain a dual graph $X^{\prime}$ is to rotate a copy of $X$ slightly in the $\phi$ and $\theta$ directions, and then isotope the stabilizing arcs of $X^{\prime}$ slightly so that they clasp those of $X$ in one to one fashion. However, we use the Morse condition on the stabilizing arcs because it allows us complete flexibility in the choice of radial edges of $X^{\prime}$ at which to base its stabilizing arcs, while still avoiding knottedness. 


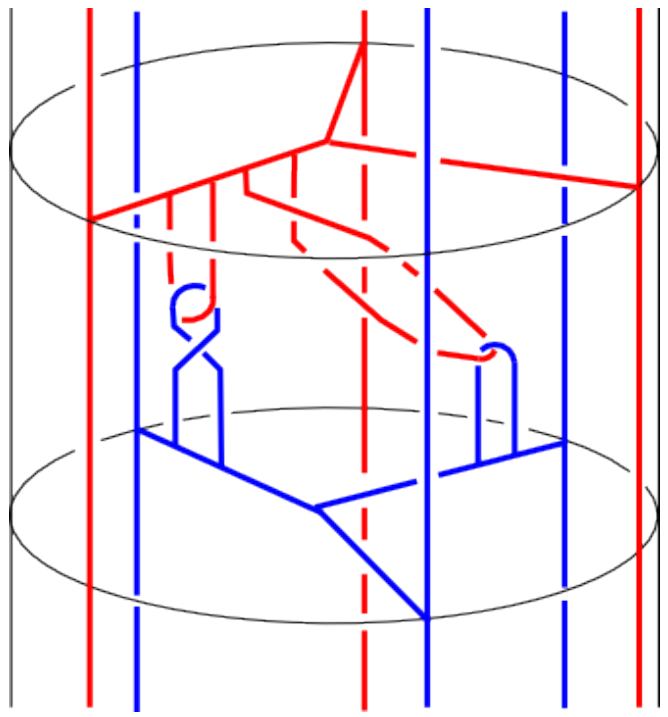

Figure 2: Dual stabilized spoke graphs.

Lemma 2.8 Let $X$ and $X^{\prime}$ be a dual pair of connected spoke surfaces in $W$, and let $B$ be the component of $E(X, W)-\stackrel{\circ}{N}\left(\mathcal{D}_{X}\right)$ that contains the central vertex of $X^{\prime}$, where $\mathcal{D}_{X}$ is the standard disk set of $X$ from Definition 2.4. Then $B$ is a ball, and $X^{\prime} \cap B$ is an unknotted tree properly embedded in $B$.

Proof It is clear that $B$ is a ball; we show that $X^{\prime} \cap B$ is unknotted. We retain the notation of Definition 2.6 throughout. If $\mathcal{C}$ is the set of stabilizing $\operatorname{arcs}$ of $X^{\prime}$, then the Morse condition on $\mathcal{C}$ ensures that $\left.h\right|_{\mathcal{C} \cap B}$ is Morse without singularities, and since $\left.h\right|_{\mathcal{D}}$ is also Morse without singularities, we can slide the endpoints of $\mathcal{C} \cap B$ off of $N(\mathcal{D}) \cap \partial B$ without introducing any further singularities. The components of $N(\mathcal{D}, W) \cap \partial B$ can then be "pushed in" so that $\partial B-\partial W$ is level with respect to $h$, and the arcs of $\mathcal{C} \cap B$ can then be properly isotoped horizontally with respect to $h$ until they are vertical. After these isotopies, it is clear that $X^{\prime} \cap B$ is unknotted.

Lemma 2.9 If $X$ and $X^{\prime}$ are connected, dual, stabilized spoke graphs, then $E(X \cup$ $\left.X^{\prime}, W\right)$ is homeomorphic to $\operatorname{Fr}(X, W) \times I$ via a map sending $\operatorname{Fr}\left(X^{\prime}, W\right)$ to $\operatorname{Fr}(X, W) \times$ $\{0\}$ and $\operatorname{Fr}(X, W)$ to $\operatorname{Fr}(X, W) \times\{1\}$.

Proof Take the double of $W$ to obtain $S^{1} \times S^{2}$, let $X_{d}$ be the double of $X$, and let $X_{d}^{\prime}$ be the double of $X^{\prime}$. Then $E\left(X_{d}, S^{1} \times S^{2}\right)$ is a handlebody since the double $\mathcal{E}$ of the standard disk set $\mathcal{D}_{X}$ cuts $E\left(X_{d}, S^{1} \times S^{2}\right)$ into balls. Moreover, $E\left(X_{d}, S^{1} \times S^{2}\right)$, $\mathcal{E}$, and $X_{d}^{\prime}$ satisfy the hypotheses of Proposition 1.14 (Lemma 2.8 handles the only 
subtle aspect of this). Thus $X_{d}^{\prime}$ is a spine of $E\left(X_{d}, S^{1} \times S^{2}\right)$, and we obtain a parameterization $E\left(X_{d} \cup X_{d}^{\prime}, S^{1} \times S^{2}\right) \cong \partial N\left(X_{d}\right) \times I$. By Proposition 1.9, the spanning annuli $(\partial W) \cap E\left(X_{d} \cup X_{d}^{\prime}, S^{1} \times S^{2}\right)$ can be assumed to be vertical with respect to this parameterization, and the result follows.

Remark 2.10 Besides being a steppingstone to Proposition 2.18 below, the significance of Lemma 2.9 is that it allows us to isotope a connected, stabilized spoke surface $S \subset W$ back and forth between small neighborhoods of dual spoke graphs lying on opposite sides of $S$ in $W$. This kind of isotopy will play an essential role in the final doppelgänger construction.

Definition 2.11 Let $\mathcal{X}$ be a disjoint union of stabilized spoke graphs embedded in $W$. Then two components $X_{1}$ and $X_{2}$ of $\mathcal{X}$ are said to be:

- $\theta$-adjacent if the closure $A$ of a component of $\overline{\partial W-N(\mathcal{X})}$ meets $N\left(X_{1}, W\right)$ in one boundary component and $N\left(X_{2}, W\right)$ in the other (in this case, $A$ is said to be a spanning annulus of $\theta$-adjacency).

- $\phi$-adjacent if there is a subarc $\beta \subset S^{1}$ such that the endpoints of $\beta \times\{0\} \subset W$ are the central vertices of $X_{1}$ and $X_{2}$, and $\beta \times\{0\}$ meets $\mathcal{X}$ only in these endpoints.

In all cases, an arc of the form $\beta \times\{0\}$ that connects the central vertices of $X_{1}$ and $X_{2}$ shall be called a spanning arc of $X_{1}$ and $X_{2}$ (there are only two such arcs).

Definition 2.12 Let $\mathcal{X}$ be a disjoint union of stabilized spoke graphs whose components are ordered $X_{0}, \ldots X_{n}$ so that $X_{i}$ is $\phi$-adjacent to $X_{i+1}$ for all $0 \leq i<n$, and let $\alpha$ be the spanning arc of $X_{0}$ and $X_{n}$ that meets all components of $\mathcal{X}$. Then $\alpha$ is said to be the binding arc of $\mathcal{X}$ with respect to the given ordering of its components.

Suppose further that, for all $1<i \leq n, X_{i}$ is $\theta$-adjacent to $X_{j_{i}}$ for some $j_{i}<i$, and let $A_{i}$ be a spanning annulus of $\theta$-adjacency connecting $X_{i}$ to $X_{j_{i}}$. Then $\mathcal{A}=$ $A_{1} \cup \cdots \cup A_{n}$ is said to form an adjacency chain for $\mathcal{X}$ with respect to the given ordering of its components.

Definition 2.13 Let $\mathcal{X}$ be a disjoint union of stabilized spoke graphs (possibly with detached longitudes in the sense of Definition 2.17 below), and let $X$ be a connected stabilized spoke graph (possibly with detached longitudes) obtained from $\mathcal{X}$ by rotating each component of $\mathcal{X}$ in the $\phi$-direction so that all of their central vertices coincide at a single vertex $x_{0}$. Then $\mathcal{X}$ is said to be a decomposition of $X$. If $d\left(x_{0}, v\right)<\epsilon$ for every central vertex $v$ occurring in a component of $\mathcal{X}$, where $d: W \times W \rightarrow \mathbb{R}$ is the flat metric, then $\mathcal{X}$ is said to be an $\epsilon$-small decomposition of $X$. 
Definition 2.14 Suppose $\mathcal{X}$ is an $\epsilon$-small decomposition of $X$ with $\epsilon<\pi / 2$. Then $\mathcal{X}$ is said to be a good decomposition of $X$ if the components $X_{0}, \ldots, X_{n}$ of $\mathcal{X}$ can be ordered so that the following conditions are satisfied:

- $X_{i}$ is $\phi$-adjacent to $X_{i+1}$ for all $1 \leq i<n$.

- The binding arc of $\mathcal{X}$ with respect to this ordering has length less than $2 \epsilon$.

- $\mathcal{X}$ admits an adjacency chain with respect to this ordering.

Observation 2.15 Let $\mathcal{X}$ be an $\epsilon$-small decomposition of $X$, and let $\alpha$ be the binding arc of $\mathcal{X}$. Then $\operatorname{Fr}(\mathcal{X} \cup \alpha, W)$ is isotopic to $\operatorname{Fr}(X, W)$, and $E(\mathcal{X}, W)$ is obtained from $E(X, W) \cong E(\mathcal{X} \cup \alpha, W)$ via 2-handle attachments to $\operatorname{Fr}(\mathcal{X} \cup \alpha, W)$ along meridians of $\alpha$.

Definition 2.16 Let $X_{1}$ and $X_{2}$ be a pair of components in $\mathcal{X}$ which are $\theta$-adjacent, with spanning annulus of $\theta$-adjacency $A$. Let $D$ be disk embedded in $W$ such that $\partial D=\alpha \cup e_{1} \cup \beta \cup e_{2}$, where $\alpha$ is a spanning arc for $X_{1}$ and $X_{2}, e_{i}$ is the radial edge of $X_{i}$ nearest to $A$ for $i=1,2, \beta \subset \partial W$ is an arc joining $e_{1}$ to $e_{2}$ that spans $A$, and $\stackrel{\circ}{D} \cap \mathcal{X}=\varnothing$. Then $D$ is said to be a spanning disk of $X_{1}$ and $X_{2}$ that cuts $A$ and is based at $\alpha$. See Figure 3 .

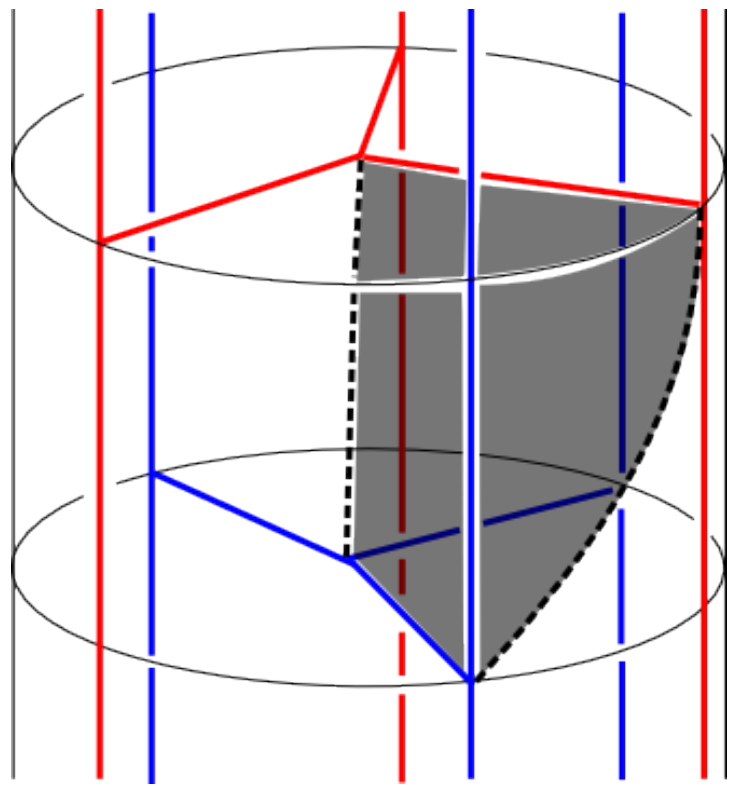

Figure 3: A spanning disk. 
Definition 2.17 Let $h_{\epsilon}: W \rightarrow W$ be the dilation $(\phi, r, \theta) \mapsto(\phi,(1-\epsilon) r, \theta)$. Let $X$ be a stabilized spoke graph, let $\mathcal{L}$ be a union of longitudinal edges of $X$, let $\mathcal{E}$ be the union of those radial edges of $X$ which meet $\mathcal{L}$, and let $\mathcal{S}$ be the union of stabilizing arcs attached to $\mathcal{E}$. Then the graph $X^{\prime}$ obtained by removing $\mathcal{E} \cup \mathcal{L} \cup \mathcal{S}$ from $X$ and attaching $h(\mathcal{E} \cup \mathcal{L} \cup \mathcal{S})$ is said to be a spoke graph obtained by $\epsilon$-small detachments of the longitudes $h(\mathcal{L})$.

In the following proposition, it is important to remember the conventions made in the final paragraph before Section 1 regarding regular neighborhoods. In particular, since $\mathcal{X}$ and $\mathcal{Y}$ are disjoint subsets of $W$, we assume their regular neighborhoods are chosen small enough so that $N(\mathcal{Y}, W) \cap N(\mathcal{X}, W)=\varnothing$.

Proposition 2.18 Suppose that $X$ and $X^{\prime}$ are dual stabilized spoke graphs embedded in $W$, that $\epsilon<\pi / 2$, and that $d\left(X, X^{\prime}\right)>\epsilon$ (as usual, $d$ is the flat metric). Suppose $\mathcal{X}$ is an $\epsilon / 8$-small, good decomposition of $X$, and that $\mathcal{A}=A_{1} \cup \cdots \cup A_{k}$ is an adjacency chain of annuli for $\mathcal{X}=X_{0} \cup \cdots \cup X_{k}$. Let $Y$ be a spoke subgraph of $X^{\prime}$ which does not meet $\mathcal{A}$, and suppose $\mathcal{Y}$ is obtained from $\epsilon / 8$-small longitudinal detachments of $Y$, followed by an $\epsilon / 8$-small decomposition (which need not be "good"). Let $\mathcal{A}$ ' be the subset of $E(\mathcal{X} \cup \mathcal{Y}, \partial W)$ consisting of those annuli which meet $\operatorname{Fr}(\mathcal{X}, W)$ on one boundary component, and $\operatorname{Fr}(\mathcal{Y}, W)$ on the other. Then $E(\mathcal{X} \cup \mathcal{Y}, W)$ is a generalized compression body $V$ such that $\partial_{v} V=\mathcal{A}^{\prime}$ and $\partial_{-} V=\operatorname{Fr}(\mathcal{Y}, W)$.

Proof We have chosen the various stabilizations and detachments small enough to ensure that they can all be carried simultaneously without creating new intersections. As in the proof of Lemma 2.9, we double $W$ to obtain $S^{1} \times S^{2}$, let $X_{d}$ denote the double of $X$, and let $X_{d}^{\prime}$ be the double of $X^{\prime}$. Then if $Y^{\prime}$ is the graph obtained by detaching some of the longitudes of $Y$, it remains isotopic to a subgraph of $X_{d}^{\prime}$ that is a spine of $E\left(X_{d}, S^{1} \times S^{2}\right)$. Thus $E\left(X_{d} \cup Y^{\prime}, S^{1} \times S^{2}\right)$ is a compression body with negative boundary $\partial N\left(Y^{\prime}\right)$.

If $\mathcal{Y}$ is any $\epsilon / 8$-small decomposition of $Y^{\prime}$, then $E\left(X_{d} \cup \mathcal{Y}, S^{1} \times S^{2}\right)$ is also a compression body because Observation 2.15 tells us that it is obtained from $E\left(X_{d} \cup\right.$ $\left.Y^{\prime}, S^{1} \times S^{2}\right)$ via 2-handle attachments along $\partial N\left(Y^{\prime}, W\right)=\partial_{-} E\left(X_{d} \cup Y^{\prime}, S^{1} \times S^{2}\right)$. The annuli $\mathcal{A}^{\prime}$ are the spanning annuli in the collection $(\partial W) \cap E\left(X_{d} \cup \mathcal{Y}, S^{1} \times S^{2}\right)$ of incompressible annuli properly embedded in $E\left(X_{d} \cup \mathcal{Y}, S^{1} \times S^{2}\right)$, and thus form the vertical boundary of the generalized compression body $E(X \cup \mathcal{Y}, W)$ cut off by $\partial W \cap E\left(X_{d} \cup \mathcal{Y}, S^{1} \times S^{2}\right)$.

Define $X_{i_{j}}$ so that each spanning annulus $A_{i}$ in our adjacency chain $\mathcal{A}$ meets $N\left(X_{i}, W\right)$ and $N\left(X_{j_{i}}, W\right)$ as in Definition 2.12. The hypothesis that $Y$ does not meet $\mathcal{A}$ implies 
the existence of a spanning disk $D_{i}$, disjoint from $\mathcal{Y}$, for each pair $X_{i}, X_{j_{i}}$. If $\alpha$ is the binding arc of $\mathcal{X}$, Observation 2.15 tells us $V^{\prime}=E(\mathcal{X} \cup \alpha \cup \mathcal{Y}, W)$ is homeomorphic to $E(X \cup \mathcal{Y}, W)$, and is thus a compression body. Let $\alpha_{i}$ be the spanning arc of $X_{i}$ and $X_{j_{i}}$ that is a subset of the binding arc $\alpha$ for $\mathcal{X}$, and let $C_{i}$ be the annulus $\operatorname{Fr}\left(\alpha_{i}, E(\mathcal{X} \cup \mathcal{Y}, W)\right) \subset \partial_{+} V^{\prime}$ (whose core curve is just a meridian of $\alpha_{i}$ ). Then the disks $\mathcal{D}=\left(D_{1} \cap V^{\prime}\right) \cup \cdots \cup\left(D_{k} \cap V^{\prime}\right)$ and annuli $\mathcal{C}=C_{1} \cup \cdots \cup C_{k}$ satisfy the hypotheses of Proposition 1.7 in $V^{\prime}$, and hence (again remembering Observation 2.15) we conclude that $E(\mathcal{X} \cup \mathcal{Y}, W)$ is a generalized compression body.

\section{The doppelgänger}

Convention 3.1 Throughout this section, $M$ is a compact, connected, orientable, irreducible 3-manifold, $\mathcal{G}$ is a generalized Heegaard surface of $M, T$ is a separating essential torus properly embedded in $M$, and $E(T, M)=M_{1} \cup M_{2}$.

Definition 3.2 $\mathcal{G}$ and $T$ are said to be well-configured with respect to $M_{1}$ if the following conditions hold:

(1) $\mathcal{G} \cap T$ consists only of simple closed curves which are essential in $T$ and $\mathcal{G}$.

(2) Each component of $\mathcal{G} \cap M_{1}$ separates $M_{1}$.

(3) For each component $V$ of $E(\mathcal{G}, M), T \cap V$ consists only of annuli which are spanning or horizontal.

Convention 3.3 For the remainder of the section, we assume that $\mathcal{G}$ and $T$ are wellconfigured with respect to $M_{1}$.

Observation 3.4 Let $\mathcal{H}=E(\mathcal{G}, M)$, which is a disjoint union of compression bodies. Let $\mathcal{A}=\operatorname{Fr}(T \cap \mathcal{H}, \mathcal{H}), \mathcal{A}^{1}=\mathcal{A} \cap M_{1}$, and $\mathcal{A}^{2}=\mathcal{A} \cap M_{2}$. Then conditions 1 and 3 of Definition 3.2, together with Proposition 1.10, imply that $\mathcal{V}=E(T \cap \mathcal{H}, \mathcal{H})$ is a generalized compression body satisfying $\partial_{v} \mathcal{V}=\mathcal{A}_{s}$, where $\mathcal{A}_{s}$ is the union of spanning annuli in $\mathcal{A}$. Moreover, if $\mathcal{A}_{h}=\mathcal{A}-\mathcal{A}_{s}$ is the subset of horizontal annuli in $\mathcal{A}$, and $\mathcal{A}_{h}^{i}=\mathcal{A}^{i} \cap \mathcal{A}_{h}$ for $i=1,2$, then there is a primitive disk set $\mathcal{D}$ for $\mathcal{V}$ with respect to some ordering of $\mathcal{A}_{h}^{1} \cup \mathcal{A}_{h}^{2}$.

Convention 3.5 The notation of Observation 3.4 is fixed for the remainder of the section. Moreover, we fix a choice of a primitive disk set $\mathcal{D}=D_{1} \cup \cdots \cup D_{n}$, which imposes the primitive orderings $\mathcal{A}_{h}^{i}=A_{1}^{i} \cup \cdots \cup A_{n}^{i}$ for $i=1,2$. Here it is understood that, for all $1 \leq j \leq n, A_{j}^{1} \cup A_{j}^{2}$ is the frontier of a single component of $T \cap \mathcal{H}$. 
Definition 3.6 Let $V$ be a component of $\mathcal{V}=E(T \cap \mathcal{H}, \mathcal{H})$. Let $\mathcal{D}^{V}=\mathcal{D} \cap V, \mathcal{A}^{V}=$ $\mathcal{A} \cap V, \mathcal{A}_{s}^{V}=\mathcal{A}_{s} \cap V=\partial_{v} V, \mathcal{A}_{h}^{V}=\mathcal{A}_{h} \cap V$, and let $\mathcal{A}_{p}^{V}$ consist of those components of $\mathcal{A}_{h}^{V}$ that are dual to some component of $\mathcal{D}^{V}$.

Lemma 3.7 For every component $V$ of $\mathcal{V}, \partial_{+} V-\mathcal{A}_{p}^{V}$ is connected.

Proof In the case $\left|\mathcal{A}_{p}^{V}\right|=0$ there is nothing to prove, so assume $\left|\mathcal{A}_{p}^{V}\right|>0$. Order the components of $\mathcal{D}^{V}=D_{1} \cup \cdots \cup D_{k}$ so that $i<j$ if $D_{i}$ has lower index than $D_{j}$ with respect to the primitive ordering of $\mathcal{D}$. Similarly, order $\mathcal{A}_{p}^{V}=A_{1} \cup \cdots \cup A_{k}$ so that $i<j$ if $A_{i}$ has lower index than $A_{j}$ with respect to the primitive ordering of $\mathcal{A}_{h}$ (so $D_{i}$ is dual to $A_{i}$ for all $1 \leq i \leq k$ ). The fact that $\mathcal{D}$ is primitive implies that $\partial D_{1}$ meets $\mathcal{A}_{p}^{V}$ only in a single spanning arc of $A_{1}$, so that $A_{1}$ meets a single component of $\overline{\partial_{+} V-\mathcal{A}_{p}^{V}}$. Likewise, $\partial D_{2}$ is disjoint from $\mathcal{A}_{p}^{V}-A_{1}$ and meets $A_{2}$ only in a single spanning arc. Since $\partial D_{2}$ does not change the component of $\partial_{+} V-\mathcal{A}_{p}^{V}$ on which it lies when it passes through $A_{1}$, it follows that $A_{2}$ also meets the same component of $\overline{\partial_{+} V-A_{p}^{V}}$ on each side. Continuing in this way for the remaining components of $\mathcal{A}_{p}^{V}$, we see that every component of $\mathcal{A}_{p}^{V}$ meets a single component of $\overline{\partial_{+} V-\mathcal{A}_{p}^{V}}$. Since $\partial_{+} V$ is connected, this implies that $\partial_{+} V-\mathcal{A}_{p}^{V}$ is also connected.

Definition 3.8 Let $V$ be a component of $\mathcal{V}$, and index the annuli $\mathcal{A}_{h}^{V}=A_{1} \cup \cdots \cup A_{m}$ so that $i<j$ implies that $A_{i}$ has lower index than $A_{j}$ with respect to the primitive ordering on $\mathcal{A}_{h}$. A set $\boldsymbol{A}=\left\{A_{i_{1}}, \ldots, A_{i_{k}}\right\}$ of components of $\mathcal{A}_{h}^{V}-\mathcal{A}_{p}^{V}$ is said to be connective if $\left(\partial_{+} V-\mathcal{A}_{h}^{V}\right) \cup A_{i_{1}} \cup \cdots \cup A_{i_{k}}$ is connected. Moreover, $\boldsymbol{A}$ is minimal if:

(1) $|\boldsymbol{A}|$ is minimal among all connective sets of $V$.

(2) For every other connective set of annuli $\boldsymbol{A}^{\prime}=\left\{A_{j_{1}}, \ldots, A_{j_{k}}\right\}$ satisfying $|\boldsymbol{A}|=$ $\left|\boldsymbol{A}^{\prime}\right|, i_{l} \leq j_{l}$ for all $1 \leq l \leq k$.

Note that conditions (1) and (2) define a unique minimal connective set with respect to any given ordering.

Convention 3.9 For the remainder of the section, let $W \cong S^{1} \times D^{2}$ be a solid torus parameterized as in Convention 2.1. Furthermore, let $T_{i}=\operatorname{Fr}(T, M) \cap M_{i}$ for $i=1,2$ and let $h: T_{1} \rightarrow \partial W$ be a homeomorphism such that each component of $h\left(\mathcal{G} \cap T_{1}\right)$ is a longitude of the form $S^{1} \times\{x\}$. Let $\pi: T_{2} \rightarrow T_{1}$ be the projection which collapses $T_{2}$ onto $T_{1}$ along the $I$-fibers of $N(T, M)$ (we assume that $\pi\left(T_{2} \cap \mathcal{G}\right)=T_{1} \cap \mathcal{G}$ ). We let $M^{\prime}=W \cup_{h \circ \pi} M_{2}$ for the remainder of the section.

Observation 3.10 If a component $V$ of $\mathcal{V} \cap M_{1}$ meets $T_{1}$ at all, then $\partial_{+} V$ must meet $T_{1}$ since the annuli of $\mathcal{A}^{V}=T_{1} \cap V$ are all either horizontal or spanning. However, it is possible that $\mathcal{A}^{V}$ consists entirely of horizontal annuli, so that $\partial_{-} V$ does not meet $T_{1}$, and this is a case which requires special treatment at certain points in our construction. 
Definition 3.11 For each component $V$ of $\mathcal{V} \cap M_{1}$ which meets $T_{1}$, let $\mathcal{B}^{V}$ denote $\overline{\partial W-h\left(\mathcal{A}^{V}\right)}$, which is a union of annuli.

Lemma 3.12 For each component $V$ of $\mathcal{V} \cap M_{1}$ which meets $T_{1}$, and each component $B$ of $\mathcal{B}^{V}$, both components of $h^{-1}(\partial B)$ lie on the same component of $\overline{\partial V-\mathcal{A}^{V}}$.

Proof If the curves of $h^{-1}(\partial B)$ lie on distinct components $F_{1}$ and $F_{2}$ of $\partial V-\mathcal{A}^{V}$, then we can construct an embedded curve in $M_{1}$ that is the union of a spanning arc $\alpha$ of $h^{-1}(B)$ and an arc $\beta$ properly embedded in $V$ with $\partial \alpha=\partial \beta$. This curve would then intersect the surface $F_{1}$ in a single point, which contradicts the assumption that each component of $\mathcal{G} \cap M_{1}$ (and hence each component of $\overline{\partial V-\mathcal{A}^{V}}$ ) is separating in $M_{1}$.

Definition 3.13 Let $V$ be a component of $\mathcal{V} \cap M_{1}$, and let $F$ be a component of $\overline{\partial V-\mathcal{A}^{V}}$ that meets $T_{1}$. Let $\mathcal{B}_{F}$ be the union of those components $B$ of $\mathcal{B}^{V}$ such that $h^{-1}(\partial B) \subset F$. If $X$ is a connected, stabilized spoke graph, possibly with detached longitudes, whose nondetached longitudinal edges are the core curves of $\mathcal{B}_{F}$, then $X$ is said to be a doppelgänger spoke graph for $F$.

Definition 3.14 Let $V$ be a component of $\mathcal{V} \cap M_{1}$ that meets $T_{1}$, and let $\mathcal{X} \cup \mathcal{Y}$ be a spoke graph constructed as follows:

(1) Let $\mathcal{B}_{+}^{V}$ be the union of those annuli in $\mathcal{B}^{V}$ that appear in $\mathcal{B}_{F}$ for some component $F$ of $\overline{\partial_{+} V-\mathcal{A}^{V}}$, let $X$ be a connected stabilized spoke graph whose longitudinal edges are the core curves of $\mathcal{B}_{+}^{V}$, and let $X^{\prime}$ be its dual. Suppose $d\left(X, X^{\prime}\right)=$ $\epsilon<\pi / 2$, where $d$ is the flat metric on $W$ as in Proposition 2.18.

(2) Suppose $\boldsymbol{A}=\left\{A_{i_{1}}, \ldots A_{i_{k}}\right\}$ is the minimal connective set of components of $\mathcal{A}_{h}^{V}-\mathcal{A}_{p}^{V}$ defined in Definition 3.8. Label the components of $\overline{\partial_{+} V-\mathcal{A}^{V}}$ that meet $A_{i_{1}}$ as $F_{0}$ and $F_{1}$, and inductively label the remaining components of $\overline{\partial_{+} V-\mathcal{A}^{V}}$ by setting $F_{j}$ equal to the component of $\overline{\partial_{+} V-\mathcal{A}^{V}}$ that meets $A_{i_{j}}$ and has not been labeled yet. At each stage of the induction, such a component of $\overline{\partial_{+} V-\mathcal{A}^{V}}$ will always exist because $\boldsymbol{A}$ was chosen to be minimal (otherwise, we could remove an element of $\boldsymbol{A}$ and still have a connective set). Moreover, since $\boldsymbol{A}$ is connective, $\overline{\partial_{+} V-\mathcal{A}^{V}}=F_{0} \cup \cdots \cup F_{k}$. Thus there exists a good $\epsilon / 8$-small decomposition $\mathcal{X}$ of $X$ such that $\mathcal{X}=X_{0} \cup \cdots \cup X_{k}$, where $X_{j}$ is a doppelgänger spoke graph of $F_{j}$. Moreover, $\mathcal{X}$ can be chosen so that $C_{i_{1}} \cup \cdots \cup C_{i_{k}}$ forms an adjacency chain for $\mathcal{X}$, where here $C_{i_{j}}$ denotes the component of $\partial W-\stackrel{\circ}{N}(X, W)$ that contains $h\left(A_{i_{j}}\right)$; see Definition 2.12. 
(3) Let $\mathcal{B}_{-}^{V}=\mathcal{B}^{V}-\mathcal{B}_{+}^{V}$, which is the union of those annuli in $\mathcal{B}^{V}$ which appear in $\mathcal{B}_{F}$ for some component $F$ of $\partial_{-} V$ that meets $T_{1}$. Every component of $\partial W-X$ contains at most one component of $\mathcal{B}_{-}^{V}$, for if two components of $\mathcal{B}_{-}^{V}$ both lie in the same component of $\partial W-X$, this would imply the existence of a component of $\mathcal{A}^{V}$ whose boundary components both lie in $\partial_{-} V$, contrary to part 3 of Definition 3.2. Thus we may assume that the core curves of $\mathcal{B}_{-}^{V}$ form a subset of the longitudinal edges of the dual $X^{\prime}$ of $X$.

(4) Define the prohibited longitudinal edges of $X^{\prime}$ to be those that lie in the same component of $\partial W-X$ as a component of $h\left(\mathcal{A}_{p}^{V}\right) \cup h\left(A_{i_{1}}\right) \cup \cdots \cup h\left(A_{i_{k}}\right)$ (there is at most one component of this set lying inside each component of $\partial W-X)$. A subgraph $Y^{\prime}$ of $X^{\prime}$ is said to be admissible if it possesses every core curve of $\mathcal{B}_{-}^{V}$ as a longitudinal edge, but no prohibited longitudinal edges.

(5) It is possible that $\mathcal{B}_{-}^{V}=\varnothing$, in which case we set $\mathcal{Y}=\varnothing$. Otherwise, let $F_{1}^{\prime}, \ldots, F_{l}^{\prime}$ be the components of $\partial_{-} V$ that meet $T_{1}$. Let $Y^{\prime}$ be an admissible subgraph of $X^{\prime}$, and let $Y$ be obtained from $Y^{\prime}$ via an $\epsilon / 8$-small detachment of those longitudes of $Y^{\prime}$ that are not core curves of $\mathcal{B}_{-}^{V}$. Finally, let $\mathcal{Y}=Y_{1} \cup \cdots \cup Y_{l}$ be an $\epsilon / 8$-small decomposition of $Y$, where $Y_{j}$ is a doppelgänger spoke graph for $F_{j}^{\prime}$ with $1 \leq j \leq l$.

Then $\mathcal{X} \cup \mathcal{Y}=X_{0} \cup \cdots \cup X_{k} \cup Y_{1} \cup \cdots \cup Y_{l}$ is said to be a doppelgänger spoke graph of $V$, and it is said to be perfect if $\operatorname{Fr}\left(X_{j}, W\right) \cong F_{j}$ and $\operatorname{Fr}\left(Y_{r}, W\right) \cong F_{r}^{\prime}$ for all $0 \leq j \leq k$ and $1 \leq r \leq l$.

Observation 3.15 The construction of Definition 3.14 was tailored to the hypotheses of Proposition 2.18. It implies that if $\mathcal{X} \cup \mathcal{Y}$ is a doppelgänger spoke graph associated with $V$, then $U=E(\mathcal{X} \cup \mathcal{Y}, W)$ is a generalized compression body satisfying $\partial_{-} U=\operatorname{Fr}(\mathcal{Y}, W)$. Then $\partial_{+} U$ is the union of $\operatorname{Fr}(\mathcal{X}, W)$ with those components of $\overline{\partial W-N(\mathcal{X} \cup \mathcal{Y}, W)}$ whose boundary components both lie in $\operatorname{Fr}(\mathcal{X}, W)$.

If $V$ does not meet $T_{1}$ as our hypotheses require, then $U$ simply does not exist. Also, if $V$ does meet $T_{1}$ but some component $F^{\prime}$ of $\partial_{-} V$ does not, then $\partial_{-} U$ will not contain a component corresponding to $F^{\prime}$.

Parts (2) and (4) of Definition 3.14 allow us to deduce the existence of a primitive disk set $\mathcal{E}^{U}$ in $U$ which will serve as a substitute for the disk set $\mathcal{D}^{V}$ of $V$, as the following lemma shows.

Lemma 3.16 Let $V$ be a component of $\mathcal{V} \cap M_{1}$ that meets $T_{1}$, let $\mathcal{X} \cup \mathcal{Y}$ be a doppelgänger spoke graph of $V$, and let $U=E(\mathcal{X} \cup \mathcal{Y}, W)$. Suppose $\mathcal{A}_{h}^{V}=A_{1} \cup$ $\cdots \cup A_{m}$ is ordered as in Definition 3.8, and that $\mathcal{A}_{p}^{V}=A_{p_{1}} \cup \cdots \cup A_{p_{q}}$. Then there is 
an ordered, disjoint collection of disks $\mathcal{E}^{U}=E_{p_{1}} \cup \cdots \cup E_{p_{q}}$ properly embedded in $U$ with the following properties:

(1) $\mathcal{E}^{U} \cap \partial_{v} U=\varnothing$.

(2) $E_{p_{j}} \cap h\left(A_{p_{j}}\right)$ is a single spanning arc for all $1 \leq j \leq q$.

(3) $E_{p_{j}} \cap h\left(A_{l}\right)=\varnothing$ for all $1 \leq j \leq q$ and $p_{j}<l \leq m$.

Proof For each component $A_{l}$ of $\mathcal{A}_{h}^{V}$, let $C_{l}$ denote the closure of the component of $\partial W-\stackrel{\circ}{N}(\mathcal{X} \cup \mathcal{Y})$ that contains $h\left(A_{l}\right)$. Let $\boldsymbol{A}=\left\{A_{i_{1}}, \ldots, A_{i_{k}}\right\}$ be the minimal connective set for $\mathcal{A}_{h}^{V}$. Order the components of $\mathcal{X}=X_{0} \cup \cdots \cup X_{k}$ as in part 2 of Definition 3.14 so that, for all $1 \leq j \leq k$, one component of $\partial C_{i_{j}}$ lies on $\operatorname{Fr}\left(X_{j}, W\right)$ and the other on some component $\operatorname{Fr}\left(X_{r}, W\right)$ of lower index $r<j$. The following technical claim is of central importance.

Claim A If $C_{p_{l}}$ meets $\operatorname{Fr}\left(X_{s}, W\right)$ and $\operatorname{Fr}\left(X_{r}, W\right)$, where $s<r$, then $i_{r}<p_{l}$.

In particular, Claim A implies that no component of $A_{p}^{V}$ lies in the minimal set $\boldsymbol{A}$, so $C_{p_{l}}$ and $C_{i_{j}}$ will be distinct for all $1 \leq l \leq q$ and $1 \leq j \leq k$.

Proof of Claim A Notice that both $C_{p_{l}}$ and $C_{i_{r}}$ connect $\operatorname{Fr}\left(X_{r}, W\right)$ to a component of $\operatorname{Fr}(\mathcal{X}, W)$ of lower index. It follows that if $p_{l}<i_{j}$, then the annulus $A_{i_{j}}$ could be replaced by $A_{p_{l}}$ in $\boldsymbol{A}$ and still yield a connective set, which violates condition 2 of Definition 3.8. If $p_{l}=i_{r}$, then $A_{p_{l}}=A_{i_{r}}$ is both a component of $A_{p}^{V}$ and a member of $\boldsymbol{A}$. As a member of the minimal set $\boldsymbol{A}$, we know that the components of $\partial A_{p_{l}}$ lie on distinct components of $\partial_{+} V-\left(\AA_{p_{l}} \cup{\stackrel{\circ}{A_{p}+1}}_{p} \cup \cdots \cup \AA_{m}\right)$. But this is incompatible with the fact that, as a component of $A_{p}^{V}$, there must be a disk properly embedded in $V$ whose boundary lies in $\partial_{+} V-\left(\stackrel{\circ}{A}_{p_{l}+1} \cup \cdots \cup \stackrel{\circ}{A}_{m}\right)$ and meets $A_{p_{l}}$ in a single spanning arc.

The remainder of the proof will mostly be devoted to the construction of a collection $K_{i_{1}} \cup \cdots \cup K_{i_{k}}$ of auxiliary "flap" disks associated with the adjacency chain $C_{i_{1}} \cup$ $\cdots \cup C_{i_{k}}$. These flap disks will then become pieces of the so-called "disks with flaps" that will form the components of $\mathcal{E}^{U}$. See Figure 4 for an example of a disk with very simple flaps.

Recalling Definitions 2.11 and 2.12, let $\alpha$ be the binding arc of $\mathcal{X}$, let $\alpha_{j}^{\prime}$ be the spanning arc of $X_{j-1}$ and $X_{j}$ that lies in $\alpha$, and let $\alpha_{j}$ denote the slightly shorter arc $U \cap \alpha_{j}^{\prime}$. Recalling Definition 2.16, let $K_{i_{1}}^{\prime}$ be the spanning disk of $X_{0}$ and $X_{1}$ that cuts $C_{i_{1}}$ and is based at $\alpha_{1}^{\prime}$. Condition 4 of Definition 3.14 ensures that $K_{i_{1}}^{\prime}$ is disjoint 


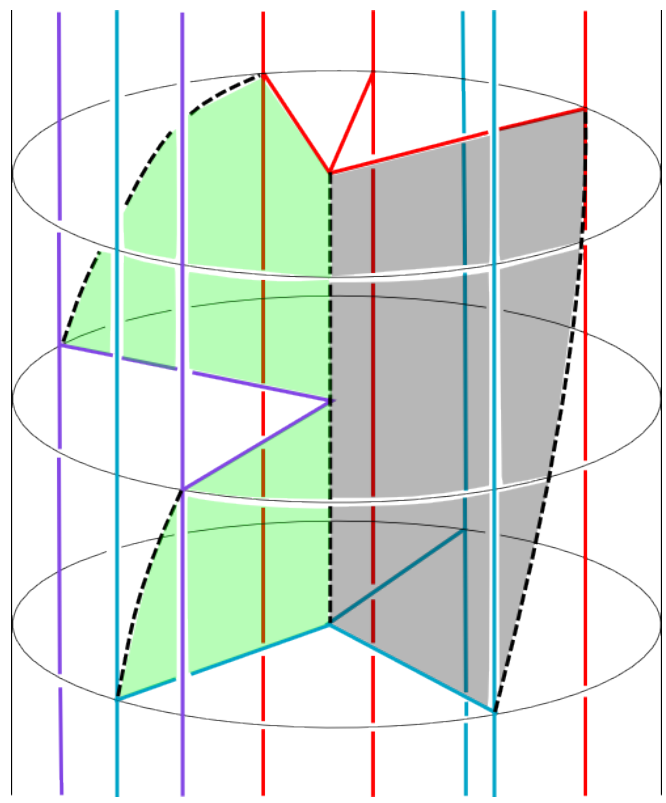

Figure 4: A disk with flaps.

from $\mathcal{Y}$. Set $K_{i_{1}}=K_{i_{1}}^{\prime} \cap U$ and observe that $\partial K_{i_{1}}=\alpha_{1} \cup \beta_{1}$, where $\beta_{1}$ is an arc lying on $\partial_{+} U$ that meets $h\left(A_{p}^{V}\right)$ only in the annulus $h\left(A_{i_{1}}\right) \subset C_{i_{1}}$.

The remaining flap disks $K_{i_{j}}$ are defined recursively. Suppose that the disks $K_{i_{s}}$ for $s<j$ have already been constructed in $U$ and satisfy the following properties:

(1) $\partial K_{i_{s}}=\alpha_{s} \cup \beta_{s}$, where $\beta_{s}$ is an arc on $\partial_{+} U$ that meets $h\left(A^{V}\right)$ only in a subset of $h\left(A_{i_{1}} \cup \cdots \cup A_{i_{s}}\right) \subset C_{i_{1}} \cup \cdots \cup C_{i_{s}}$.

(2) $K_{i_{s}}$ meets $\alpha \cap U$ only in $\alpha_{s}$.

(3) $K_{i_{s}} \cap K_{i_{r}}=\varnothing$ for all $r<s<j$.

We will construct $K_{i_{j}}$ so that it also satisfies these properties. To begin with, we know that $C_{i_{j}}$ meets $\operatorname{Fr}\left(X_{j}, W\right)$ and $\operatorname{Fr}\left(X_{r}, W\right)$ for some $r<j$. Let $D$ be the spanning disk for $X_{r}$ and $X_{j}$ that cuts $C_{i_{j}}$ and is based at $\alpha_{r+1}^{\prime} \cup \cdots \cup \alpha_{j}^{\prime}$. If we construct the disks $K_{i_{s}}$ correctly in the "obvious" way (or rather, the way that is most directly imaginable after some careful thought), then $K=K_{i_{r+1}} \cup \cdots \cup K_{i_{j-1}} \cup(D \cap U)$ will already be an embedded disk in $U$ satisfying property 1 , and after a small isotopy it could be made to satisfy 2 and 3 as well (in the case that $r=j-1$ we just set $K=D \cap U)$. A formal description of these so-called "obvious" disks would be rather tedious and obscure, so we will take a more abstract route in the description of $K_{i_{j}}$. However, it is a helpful exercise to visualize what $K_{i_{2}}$ could look like. 
As it stands, our hypotheses on the disks $K_{i_{s}}$ allow the possibility that $D \cap U$ meets $\mathcal{K}=K_{i_{r+1}} \cup \cdots \cup K_{i_{j-1}}$ not only along $\alpha_{r+1} \cup \cdots \cup \alpha_{j-1}$, which is desirable, but also (transversely, as always) along other "undesirable" arcs and simple closed curves disjoint from $\alpha_{r+1} \cup \cdots \cup \alpha_{j}$. We eliminate the undesirable intersections as follows. First, the simple closed curves of intersection can be eliminated via isotopy of $D \cap U$ in $U$. If $D \cap U$ meets $\mathcal{K}$ along any undesirable arcs, then it must meet some component $K_{i_{s}}$ of $\mathcal{K}$ along an undesirable arc $\gamma$ which bounds a disk $\delta \subset K_{i_{s}}$ with the following properties:

- $\partial \delta \subset \gamma \cup\left(\partial K_{i_{s}}-\alpha_{s}\right)$.

- $\stackrel{\circ}{\delta} \cap D=\varnothing$.

In other words, $\delta$ is an outermost disk of intersection whose boundary does not contain the $\operatorname{arc} \alpha_{s}$. If $D^{\prime \prime}$ is the component of $(D \cap U)-\gamma$ that contains $\alpha_{j}$ in its boundary, $D^{\prime}=D^{\prime \prime} \cup \delta$ is a disk which can be isotoped slightly away from $\gamma$ so that it intersects $\mathcal{K}$ in strictly fewer arcs than $D \cap U$. It is also possible that $D^{\prime}$ contains fewer of the $\operatorname{arcs} \alpha_{s}$ for $r<s<j$, so let $\mathcal{K}^{\prime}$ denote the union of those $K_{i_{s}}$ that meet $D^{\prime \prime}$ along $\alpha$.

Certainly $D^{\prime}$ meets $\mathcal{K}^{\prime}$ in fewer undesirable arcs than $D$ met $\mathcal{K}$. Thus, by repeating this process, we eventually will obtain a disk (call it $D^{\prime}$ as well) that still contains $\alpha_{j}$ in its boundary, as well as a corresponding subset of components of $\mathcal{K}$ (call it $\mathcal{K}^{\prime}$ as well) such that $D^{\prime}$ meets $\mathcal{K}^{\prime}$ only along desirable arcs. Then $D^{\prime} \cup \mathcal{K}^{\prime}$ will be a disk embedded in $U$ that satisfies property 1 above and, after a small isotopy, property 2 as well. If necessary, another round of outermost disk surgeries that preserve the piece of $D^{\prime}$ that contains $\alpha_{j}$ can eliminate any intersections of $D^{\prime} \cup \mathcal{K}^{\prime}$ with $K_{i_{1}} \cup \cdots \cup K_{i_{j-1}}$. The result is the desired disk $K_{i_{j}}$ that satisfies property 3 as well.

We are now ready to construct the disks $E_{p_{j}}$. The first kind we construct are those with index $p_{j}$ such that both components of $\partial C_{p_{j}}$ lie on a single component $\operatorname{Fr}\left(X_{r}, W\right)$ of $\operatorname{Fr}(\mathcal{X}, W)$, where $X_{r}$ has central vertex $v=\left(\phi_{0}, 0,0\right)$. In the notation of part 4 of Definition 3.14, $Y^{\prime}$ must be disjoint from $C_{p_{j}}$ since the longitude of $X^{\prime}$ that lies in $C_{p_{j}}$ is prohibited. Hence $\mathcal{Y}$ is disjoint from the component of $\left(\left\{\phi_{0}\right\} \times D^{2}\right) \cap E(\mathcal{X}, W)$ that meets $C_{p_{j}}$. We define $E_{p_{j}}$ to be this component, whose boundary lies in $\partial_{+} U$ and meets $h\left(\mathcal{A}^{V}\right)$ only in a single spanning arc of $A_{p_{j}}$. Hence it satisfies conclusions (1), (2), and (3) of our lemma.

The remaining components of $\mathcal{E}^{U}$ will be "disks with flaps"; see Figure 4 for a simple example. Such disks have indexes $p_{j}$ such that the components of $\partial C_{p_{j}}$ lie on distinct components $\operatorname{Fr}\left(X_{s}, W\right)$ and $\operatorname{Fr}\left(X_{r}, W\right)$ of $\operatorname{Fr}(\mathcal{X}, W)$ with $s<r$. Let $D$ be the spanning disk of $X_{s}$ and $X_{r}$ that cuts $C_{p_{j}}$ and is based on the spanning arc $\alpha_{s+1}^{\prime} \cup \cdots \cup \alpha_{r}^{\prime}$ of $X_{s}$ and $X_{r}$. Then $D \cap U$ will meet $\mathcal{K}=K_{i_{s+1}} \cup \cdots \cup K_{i_{r}}$ along "desirable" $\operatorname{arcs} \alpha_{s+1} \cup \cdots \cup \alpha_{r}$, and possibly also along undesirable simple closed curves 
and arcs, which form the self intersections of the immersed disk $E^{\prime}=(D \cap U) \cup \mathcal{K}$ whose boundary lies entirely on $\partial_{+} U$. Moreover, $D \cap U$ meets $h\left(\mathcal{A}^{V}\right)$ only in $A_{p_{j}}$, and Claim A and property 1 of the flap disks ensure $\mathcal{K}$ can only meet $h\left(\mathcal{A}^{V}\right)$ in components of the form $h\left(A_{l}\right)$ with $l<p_{j}$. Hence the boundary of $E^{\prime}$ already has the desired properties. As with the construction of the flap disks, we can now eliminate the self-intersections of $E^{\prime}$ by performing isotopies and a sequence of outermost disk surgeries on $D \cap U$ that preserve its intersection with $h\left(A_{p_{j}}\right)$. The result is our desired "disk with flaps" $E_{p_{j}}$ that does satisfy conclusions (1)-(3) of our lemma.

One technicality to note is that, as constructed, it is possible for some of the components of $\mathcal{E}^{U}$ to intersect, but again a sequence of appropriate innermost/outermost disk surgeries can be employed to turn it into a disjoint union of disks with the desired properties if necessary.

Proposition 3.17 For every component $V$ of $\mathcal{V} \cap M_{1}$ that meets $T_{1}$, there is a perfect doppelgänger spoke graph embedded in $W$.

Proof For any doppelgänger spoke graph $X_{F}$ of a component $F$ of $\overline{\partial V-\mathcal{A}^{V}}$, the surface $\operatorname{Fr}\left(X_{F}, W\right)$ will have the same number of boundary components as $F$. Moreover, the genus of $\operatorname{Fr}\left(X_{F}, W\right)$ is the same as the total number of stabilizing arcs and detached longitudes that occur in $X_{F}$. In particular, if $X_{F}$ is unstabilized and has no detached longitudes, then $\operatorname{Fr}\left(X_{F}, W\right)$ will be planar. Thus we can always find a doppelgänger spoke graph $X_{F}$ that satisfies $\operatorname{Fr}\left(X_{F}, W\right) \cong F$ after attaching a sufficient number of stabilizing arcs and/or detached longitudes.

In part 1 of Definition 3.14, we have the flexibility to stabilize $X$ as often as we need, with stabilizing arcs based on radial edges of our choosing. This allows us to choose the number of stabilizing arcs that will eventually occur in the components of the spoke graph $\mathcal{X}$ defined in part 2 of Definition 3.14. It follows from this and the previous paragraph that we may choose $\mathcal{X}$ so that each of its components $X_{j}$ satisfies $\operatorname{Fr}\left(X_{j}, W\right) \cong F_{j}$.

As noted in Remark 2.7, the Morse condition of Definition 2.6 grants us enough flexibility to choose the radial edges on which the stabilizing arcs of $X^{\prime}$ (the dual of $X$ ) will be based, and this in turn allows us to control the component of $\mathcal{Y}$ on which they will eventually occur in part 5 of Definition 3.14. Likewise, we can choose the components of $\mathcal{Y}$ on which the detached longitudes of $Y$ shall occur after the decomposition described in part 5 of Definition 3.14.

So, as in the case with $\mathcal{X}$, we may distribute detached longitudes and stabilizing arcs among the components of $\mathcal{Y}$ however we please. But there is an important difference: 
the total number of stabilizing arcs and detached longitudes that can occur in $\mathcal{Y}$ is bounded above by $s+a$, where $s$ denotes the number of stabilizing arcs that occur in $X$, and $a$ denotes the maximal number of detached longitudes that can occur on an admissible subgraph of $X^{\prime}$ (as defined in Definition 3.14(4)). Therefore, to complete the proof, we must show that a total of $s+a$ stabilizing arcs and detached longitudes is always sufficient to create a spoke graph $\mathcal{Y}$ that satisfies the equation $\operatorname{Fr}\left(Y_{j}, W\right) \cong F_{j}^{\prime}$ for each of its components $Y_{j}$.

As in Definition 3.14(5), let $\mathcal{F}^{\prime}=F_{1}^{\prime} \cup \cdots \cup F_{l}^{\prime}$ be the union of those components of $\partial_{-} V$ that meet $T_{1}$. By the first paragraph of this proof, the total number of stabilizing arcs and detached longitudes necessary to ensure that $\operatorname{Fr}\left(Y_{j}, W\right) \cong F_{j}^{\prime}$ for all $1 \leq j \leq l$ is equal to $\sum g\left(F_{j}^{\prime}\right)$, where $g\left(F_{j}^{\prime}\right)$ is the genus of $F_{j}^{\prime}$. Since $g(F)=1-\frac{1}{2} \chi(F)+|\partial F|$ for any connected, compact surface $F$, we obtain

$$
\sum g\left(F_{j}^{\prime}\right)=\left|\mathcal{F}^{\prime}\right|-\frac{\chi\left(\mathcal{F}^{\prime}\right)+\left|\mathcal{A}_{s}^{V}\right|}{2} .
$$

The fact that this quantity is less than $s+a$ is ultimately derived from the inequality

$$
\chi\left(\partial_{+} V\right)-\chi\left(\partial_{-} V\right) \leq-2\left(\left|\mathcal{A}_{p}^{V}\right|+\left|\partial_{-} V\right|-1\right) .
$$

The truth of (2) can be seen as follows: $V^{\prime}=E\left(\mathcal{D}^{V}, V\right)$ is a generalized compression body with the same negative boundary as $V$. Furthermore, $\partial_{+} V^{\prime}$ is connected since $\partial_{+} V-\partial \mathcal{D}^{V}$ is connected, as can be seen using essentially the same proof as that of Lemma 3.7. Now $\partial_{-} V^{\prime}$ is obtained from $\partial_{+} V^{\prime}$ via surgeries along disks, and there must be at least $\left|\partial_{-} V^{\prime}\right|-1$ such surgeries since $\partial_{+} V^{\prime}$ is connected. Thus $\chi\left(\partial_{+} V^{\prime}\right)-\chi\left(\partial_{-} V^{\prime}\right) \leq-2\left(\left|\partial_{-} V^{\prime}\right|-1\right)$. Inequality (2) now follows from the fact that $\partial_{-} V^{\prime}=\partial_{-} V$, and the fact that $\chi\left(\partial_{+} V^{\prime}\right)=\chi\left(\partial_{+} V\right)+2\left|\mathcal{D}^{V}\right|=\chi\left(\partial_{+} V\right)+2\left|\mathcal{A}_{p}^{V}\right|$.

Since no component of $\partial_{-} V$ is a disk or sphere, and since $\mathcal{F}^{\prime} \subset \partial_{-} V$, we have $\chi\left(\partial_{-} V\right) \leq \chi\left(\mathcal{F}^{\prime}\right)$. Thus, from (2), we easily obtain the analogue $\chi\left(\partial_{+} V\right)-\chi\left(\mathcal{F}^{\prime}\right) \leq$ $-2\left(\left|\mathcal{A}_{p}^{V}\right|+\left|\mathcal{F}^{\prime}\right|-1\right)$. In conjunction with equation (1), we obtain

$$
\sum g\left(F_{j}^{\prime}\right) \leq 1-\left|\mathcal{A}_{p}^{V}\right|-\frac{\chi\left(\partial_{+} V\right)+\left|\mathcal{A}_{s}^{V}\right|}{2} .
$$

Our choice of $\mathcal{X}$ has ensured that $\chi\left(\partial_{+} V\right)=\chi(\operatorname{Fr}(\mathcal{X}, W))$. We then compute $\chi(\operatorname{Fr}(\mathcal{X}, W))=-|\partial \operatorname{Fr}(\mathcal{X}, W)|-2 s+2|\operatorname{Fr}(\mathcal{X}, W)|=-2\left|\mathcal{A}_{h}^{V}\right|-\left|\mathcal{A}_{s}^{V}\right|-2 s+2|\mathcal{X}|$, and so deduce

$$
\sum g\left(F_{j}^{\prime}\right) \leq 1-\left|\mathcal{A}_{p}^{V}\right|+\left|\mathcal{A}_{h}^{V}\right|+s-|\mathcal{X}| .
$$

The detached longitudes of $Y$, as described in part 5 of Definition 3.14, all come from core curves of the annuli of $\partial W-X$ that contain a component of $h\left(\mathcal{A}_{h}^{V}\right)$. On the 

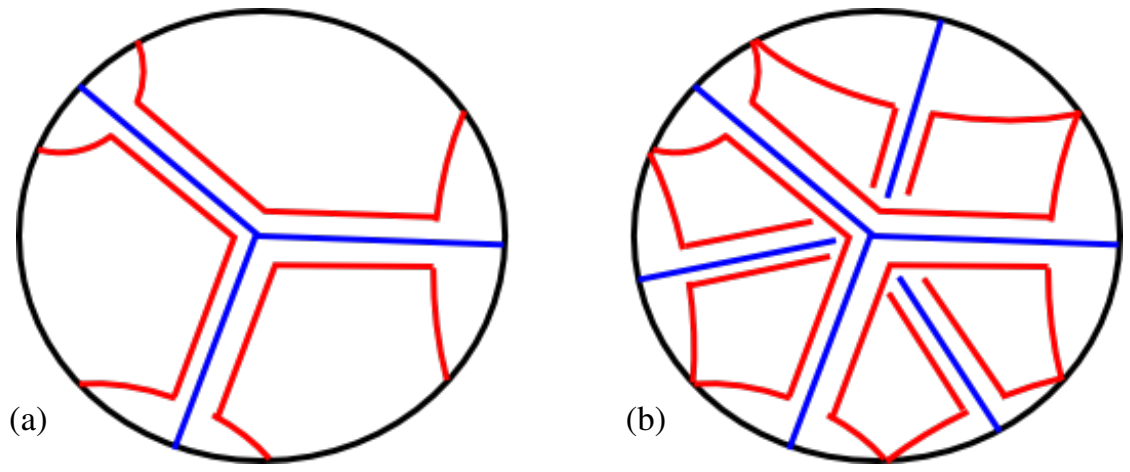

Figure 5: On the left, an image of a component $X$ of $\mathcal{X} \cup \mathcal{Y}$ of Definition 3.18 in blue, projected onto the meridian disk of $W$. We have chosen $\operatorname{Fr}(X, W)$, in red, to lie close to $X$ in the usual way near the radial edges, but it spreads out near $\partial W$ to meet $h\left(\partial \mathcal{G}^{V}\right)$. On the right, a spoke graph $Y$ and its dual $Y^{\prime}$ (both in blue), together with $\operatorname{Fr}(Y, W)$ and $\operatorname{Fr}\left(Y^{\prime}, W\right)$, chosen as in Lemma 3.20.

other hand, the number of prohibited annuli (part 4 of Definition 3.14) is equal to $\left|\mathcal{A}_{p}^{V}\right|+|\boldsymbol{A}|$, where $\boldsymbol{A}$ denotes the set of annuli defined in part 2 of Definition 3.14. Hence there will be at most $a=\left|\mathcal{A}_{h}^{V}\right|-\left|A_{p}^{V}\right|-|\boldsymbol{A}|$ detached longitudes which may occur in $\mathcal{Y}$. However, $|\boldsymbol{A}|=|\mathcal{X}|-1$ by the minimality of $|\boldsymbol{A}|$. Plugging this into inequality (4) yields

$$
\sum g\left(F_{j}^{\prime}\right) \leq s+a
$$

The proposition now follows.

Definition 3.18 Let $V$ be a component of $\mathcal{V} \cap M_{1}$ that meets $T_{1}$, let $\mathcal{G}^{V}$ denote the union of those components $G$ of $\mathcal{G} \cap M_{1}$ such that $N\left(G, M_{1}\right) \cap V \neq \varnothing$ and $G \cap T_{1} \neq \varnothing$. Let $\mathcal{X} \cup \mathcal{Y}$ be a perfect doppelgänger spoke graph for $V$, and let $\mathcal{Q}^{V}=\operatorname{Fr}(\mathcal{X} \cup \mathcal{Y}, W)$ where we have chosen $\operatorname{Fr}(\mathcal{X} \cup \mathcal{Y}, W)$ so that $\partial \operatorname{Fr}(\mathcal{X} \cup \mathcal{Y}, W)=h\left(\partial \mathcal{G}^{V}\right)$ as in Figure 5(a). Then $\mathcal{Q}^{V}$ is called the doppelgänger surface of $V$, and the closure of the component of $W-\mathcal{Q}^{V}$ that does not contain $\mathcal{X} \cup \mathcal{Y}$ is the doppelgänger chamber of $V$.

Remark 3.19 Suppose now that two components $V$ and $V^{\prime}$ of $\mathcal{V} \cap M_{1}$ that both meet $T_{1}$ are adjacent in the obvious sense, which means that $N\left(G, M_{1}\right)$ meets both $V$ and $V^{\prime}$ for some component $G$ of $\mathcal{G} \cap M_{1}$ that meets $T_{1}$. If $Q$ is the component of $\mathcal{Q}^{V}$ corresponding to $G$, and $Q^{\prime}$ is the component of $\mathcal{Q}^{V^{\prime}}$ corresponding to $G$, then $\partial Q=\partial Q^{\prime}$. Identifying $Q$ with $\operatorname{Fr}(Y, W)$ and $Q^{\prime}$ with $\operatorname{Fr}\left(Y^{\prime}, W\right)$ in Lemma 3.20 below, we see that in fact $Q$ is isotopic to $Q^{\prime}$ via an isotopy which fixes $\partial Q$. Moreover, 
the proof will show that this isotopy, performed ambiently, will push the doppelgänger chamber $U$ of $V$ into the side of $Q^{\prime}$ that does not contain the doppelgänger chamber $U^{\prime}$ of $V^{\prime}$. We shall call such an isotopy a flipping isotopy.

Lemma 3.20 Let $X$ be a connected, unstabilized spoke graph and let $X^{\prime}$ be its dual. Suppose $Y$ is obtained from $X$ by attaching a total of $n$ stabilizing arcs and detached longitudes, and that $Y^{\prime}$ is obtained from $X^{\prime}$ by attaching a total of $n$ stabilizing arcs and detached longitudes, in any fashion. Then if we choose $\operatorname{Fr}(Y, W)$ and $\operatorname{Fr}\left(Y^{\prime}, W\right)$ so that their boundaries coincide as in Figure 5(b), $\operatorname{Fr}(Y, W)$ will be isotopic to $\operatorname{Fr}\left(Y^{\prime}, W\right)$ via an isotopy which remains fixed on $\partial W$.

Proof If $Y$ has detached longitudes, then there is a sequence of edge slides that change $Y$ into a connected stabilized spoke graph $\tilde{Y}$ without detached longitudes (see Figure 6), and these correspond to an isotopy of $\operatorname{Fr}(Y, W)$ to $\operatorname{Fr}(\tilde{Y}, W)$, one which we can choose to be supported outside of a small open collar of $\partial W$.

If $\tilde{Y}^{\prime}$ is the dual of $\tilde{Y}$, and we choose $\operatorname{Fr}\left(\tilde{Y}^{\prime}, W\right)$ so that its boundary coincides with the boundary of $\operatorname{Fr}(\tilde{Y}, W)$ (as in Figure 5(b)), then Lemma 2.9 (see also Remark 2.10) tells us that the region of $W$ trapped between $\operatorname{Fr}\left(\tilde{Y}^{\prime}, W\right)$ and $\operatorname{Fr}(\tilde{Y}, W)$ can be parameterized as a "pinched" thickened surface whose vertical boundary has been collapsed. Hence we can isotope $\operatorname{Fr}(\tilde{Y}, W)$ onto $\operatorname{Fr}\left(\tilde{Y}^{\prime}, W\right)$ via an isotopy that is fixed on $\partial W$.

Finally, since $\tilde{Y}^{\prime}$ is obtained from $X^{\prime}$ by attaching $n$ stabilizing arcs, using (a reversed version of) the same kind of isotopy described in the first paragraph, we may slide the stabilizing arcs of $\tilde{Y}^{\prime}$ along $X^{\prime}$ so that they coincide with the stabilizing arcs and detached longitudes of $Y^{\prime}$. This corresponds to an isotopy of $\operatorname{Fr}\left(\tilde{Y}^{\prime}, W\right)$ onto $\operatorname{Fr}\left(Y^{\prime}, W\right)$ that is fixed on $\partial \operatorname{Fr}\left(\tilde{Y}^{\prime}, W\right)$.

Theorem 3.21 If the generalized Heegaard surface $\mathcal{G} \subset M$ amalgamates to a minimal genus Heegaard surface of $M$, then $g\left(M^{\prime}\right) \leq g(M)$ (here $M^{\prime}$ is the manifold obtained by gluing $W$ to $M_{2}$ as described in Convention 3.9).

Proof Our assumption that $T$ and $\mathcal{G}$ are well-configured does not eliminate the possibility that $T \cap \mathcal{G}=\varnothing$. But in this case, $T$ will be parallel to a component of $\mathcal{G}_{-}$, the thin part of $\mathcal{G}$, which implies the stronger conclusion $g\left(M_{1}\right)+g\left(M_{2}\right)=g(M)$. So we assume $T \cap \mathcal{G} \neq \varnothing$.

Suppose that $V_{1} \cup \cdots \cup V_{n}$ is the union of all components of $\mathcal{V} \cap M_{1}$ that meet $T_{1}$, and let $\left\{U_{1}, \ldots, U_{n}\right\}$ be the corresponding set of doppelgänger chambers in $W$. If the $U_{i}$ are embedded exactly as in Definition 3.18, then they will intersect one another badly. However, using Lemma 3.20 (and Remark 3.19 preceding it) we can show: 


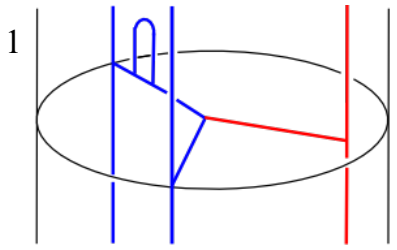

2
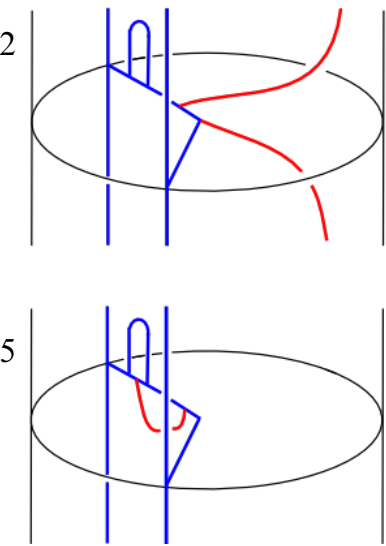

3

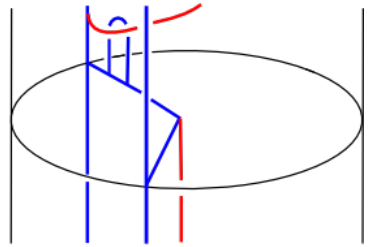

6

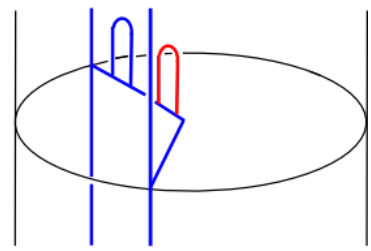

Figure 6: Edge slides that turn a detached longitude into a stabilized arc.

Claim B The $U_{i}$ can be isotoped in $W$, via isotopies which leave $\partial W$ fixed, so that:

(1) $U_{1} \cup \cdots \cup U_{n}=W$.

(2) For all $i \neq j, U_{i} \cap U_{j}=\varnothing$ unless $V_{i}$ is adjacent to $V_{j}$ as in Remark 3.19.

(3) If $V_{i}$ is adjacent to $V_{j}$, then $U_{i} \cap U_{j}=\left(\partial_{+} U_{i} \cap \partial_{+} U_{j}\right) \cup\left(\partial_{-} U_{i} \cap \partial_{-} U_{j}\right)$, and it is a single component of $\partial_{ \pm} U_{i}$.

We shall prove Claim B at the end. Assuming it is established, let $\mathcal{Q}$ denote the union of all surfaces of the form $U_{i} \cap U_{j}$ with $i \neq j$, which is the same as the union of all surfaces $\partial_{+} U_{i} \cup \partial_{-} U_{i}$. Moreover, for all $1 \leq i \leq n$, let $\tilde{U}_{i}$ be the component of $E(\mathcal{Q}, W)$ corresponding to $U_{i}$. Thus $\tilde{U}_{i}$ is just the result of carving out a collar of $\partial_{+} U_{i} \cup \partial_{-} U_{i}$ from $U_{i}$; we need it here so that it will match up snugly with the components of $\mathcal{V} \cap M_{2}$, but morally it should just be thought of as $U_{i}$. The following facts are now easily verified for all $1 \leq i \leq n$ :

- $h\left(\partial_{v} V_{i}\right)=\partial_{v} \tilde{U}_{i}$

- $h\left(\partial_{+} V_{i} \cap T_{1}\right)=\partial_{+} \tilde{U}_{i}$.

Hence by Observation 1.2, the result of attaching the vertical annuli of $\left(\mathcal{V} \cap M_{2}\right)$ to the vertical annuli of $\tilde{U}_{1} \cup \cdots \cup \tilde{U}_{n}$ along $h \circ \pi$ will be a generalized compression body, and it will have no vertical boundary components (they have all been glued together).

Moreover, if $\mathcal{E}$ is the union of all the (deformed versions of) the disk sets $\mathcal{E}^{U_{i}}$ defined in Lemma 3.16, then Lemma 3.16 implies that $\mathcal{E} \cup\left(\mathcal{D} \cap M_{2}\right)$ admits an ordering which makes it a primitive disk set for the ordered union of annuli $h\left(\mathcal{A}_{h}^{1}\right) \cup \mathcal{A}_{h}^{2}$ (see Definition 1.4 and Convention 3.5) with respect to the map $h \circ \pi$ (see Convention 3.9). 
By Proposition 1.6, the result of gluing $E(\mathcal{Q}, W)=\tilde{U}_{1} \cup \cdots \cup \tilde{U}_{n}$ to $E\left(\mathcal{G}, M_{2}\right)=\mathcal{V} \cap M_{2}$ along $h \circ \pi$ is a generalized compression body. But this is just $E\left(\mathcal{Q} \cup_{h \circ \pi}\left(\mathcal{G} \cap M_{2}\right), M^{\prime}\right)$. Hence $\mathcal{G}^{\prime}=\mathcal{Q} \cup_{h \circ \pi}\left(\mathcal{G} \cap M_{2}\right)$ is a generalized Heegaard surface in $M^{\prime}$. The surface $\mathcal{G}^{\prime}$ may have fewer components than $\mathcal{G}$, but in any event it will amalgamate to a surface of genus less than or equal to the genus of the surface that $\mathcal{G}$ amalgamates to in $M$. Thus we will be finished once we prove Claim B.

Proof of Claim B We will describe an algorithm which uses flipping isotopies to embed the $U_{i}$ in the desired fashion. Start by embedding $U_{1}$ as described in Definition 3.18. Suppose now that $V_{1}$ is adjacent to $V_{i}$ along some component $G$ of $\mathcal{G} \cap M_{1}$ (such a component $G$ will be unique by Convention 3.3 above), which means that $N\left(G, M_{1}\right)$ meets $V_{1}$ and $V_{i}$ in components $F_{1}$ of $\partial_{ \pm} V_{1}$ and $F_{i}$ of $\partial_{ \pm} V_{i}$. If $R_{1}$ and $R_{i}$ are the corresponding components of $\partial_{ \pm} U_{1}$ and $\partial_{ \pm} U_{i}$, then Remark 3.19 and Lemma 3.20 tell us that $R_{1}$ can be isotoped onto $R_{i}$ via a flipping isotopy which is fixed on $\partial W$. Moreover, this flipping isotopy, if performed ambiently, will push $U_{1}$ into $\overline{W-U_{i}}$, where $U_{i}$ is now embedded as described in Definition 3.18 (and $U_{1}$ is now distorted).

Now suppose $V_{1}$ is adjacent to another component $V_{j}$ of $\mathcal{V} \cap M_{1}$ along a component $G^{\prime}$ of $\mathcal{G} \cap M_{1}$, and suppose $R_{1}^{\prime}$ and $R_{j}^{\prime}$ are the components of $\partial_{ \pm} U_{1}$ and $\partial_{ \pm} U_{j}$, respectively, that correspond to $G^{\prime}$. Reverse the flipping isotopy of the previous paragraph, so that $U_{1}$ will again be embedded in the standard way, and $U_{i}$ will be distorted. We can then perform a further flipping isotopy taking $R_{1}^{\prime}$ to $R_{j}^{\prime}$, which will push $U_{1}$ and the distorted version of $U_{i}$ into $\overline{W-U_{j}}$, where we assume $U_{j}$ is now embedded in the standard way.

Repeating the process of the previous two paragraphs, we can eventually embed (distorted versions of) the doppelgänger chambers of every other component of $\mathcal{V} \cap M_{1}$ that is adjacent to $V_{1}$ in a way that satisfies part 2 of Claim B. Once this is done, we can then embed the components of $\mathcal{V} \cap M_{1}$ that are adjacent to those components of $\mathcal{V} \cap M_{1}$ adjacent to $V_{1}$, and so on. Eventually this process will terminate, and we will have embedded every doppelgänger $U_{i}$ of every component of $\mathcal{V} \cap M_{1}$ that meets $T_{1}$, and it is easy to verify that part 1 of Claim B will then be satisfied as well. The claim and theorem now follow.

Corollary 3.22 If $\mathcal{G}$ amalgamates to a minimal genus Heegaard surface of $M$, then $g\left(M_{2}\right) \leq g(M)+1$.

Proof Since the core $c$ of $W$ can be embedded in the surface $\mathcal{G}^{\prime}$ constructed in the proof of Theorem 3.21, we can stabilize $\mathcal{G}^{\prime}$ once (if necessary) to obtain a generalized Heegaard splitting of $E\left(c, M^{\prime}\right) \cong M_{2}$ of genus at most $g(M)+1$. 


\section{The main result}

Theorem 4.1 If $K_{1}$ and $K_{2}$ are knots in $S^{3}$, then $t\left(K_{1} \# K_{2}\right) \geq \max \left\{t\left(K_{1}\right), t\left(K_{2}\right)\right\}$.

Proof The proposition is trivial if one of $K_{1}$ or $K_{2}$ is the unknot, so suppose that both are nontrivial knots. Assume also that $\max \left\{t\left(K_{1}\right), t\left(K_{2}\right)\right\}=t\left(K_{2}\right)$. Let $T$ be the "swallow-follow" torus in $E\left(K_{1} \# K_{2}\right)$ that swallows the $K_{2}$ summand and follows the $K_{1}$ summand; see Figure 7. We apply Theorem 3.21 by setting $M=E\left(K_{1} \# K_{2}\right)$, noting that one component of $E(T, M)$ is homeomorphic to $E\left(K_{1}\right)$, which will correspond to $M_{1}$. What needs to be shown is that the untelescoped minimal splitting $\mathcal{G}$ can be isotoped so that it meets $T$ only in essential simple closed curves, and such that each component of $\mathcal{G} \cap E\left(K_{1}\right)$ is separating.

By its definition as a swallow-follow torus, $T$ is isotopic to $A \cup B$, where $A$ is the decomposing annulus of the connected sum in $M=E\left(K_{1} \# K_{2}\right)$, and $B$ is the subannulus of $\partial M-A$ that lies in the component of $M-A$ corresponding to $E\left(K_{1}\right)$. By Proposition 1.21, $\mathcal{G}$ can be isotoped to intersect $A$ only in essential simple closed curves, and since each boundary component of $\mathcal{G} \cap E\left(K_{1}\right)$ is then a standard meridional curve of $\partial E\left(K_{1}\right)$, every component of $\mathcal{G} \cap E\left(K_{1}\right)$ is separating in $E\left(K_{1}\right)$ (otherwise we could obtain a nonseparating surface in $S^{3}$ ). The hypotheses of Theorem 3.21 (which assume Conventions 3.1, 3.3, and 3.9) can then be satisfied by isotoping $T$ sufficiently close to $A \cup B$.

Now $M_{2}$ is the component of $E(T, M)$ which is not homeomorphic to $E\left(K_{1}\right)$, but is instead homeomorphic to $E(L)$, where $L$ is the link in $S^{3}$ that has $K_{2}$ as one component and a meridian $\mu$ of $K_{2}$ as its other component, and $T=\partial N(\mu)$ under this correspondence; see Figure 7. Furthermore, the slope in which $\mathcal{G}$ has been made to intersect $T=\partial N(\mu)$ is the standard longitudinal slope determined by the meridian disk $\delta \subset S^{3}$ with $\partial \delta=\mu$ and $\left|\delta \cap K_{2}\right|=1$. Thus the slope of the trivial Dehn filling of $\partial N(\mu)=T$ that yields $E\left(K_{2}\right)$ meets each component of $T \cap \mathcal{G}$ exactly once, and Theorem 3.21 applies to $M^{\prime}=E\left(K_{2}\right)$, yielding

$$
t\left(K_{1} \# K_{2}\right)=g\left(E\left(K_{1} \# K_{2}\right)\right)-1 \geq g\left(E\left(K_{2}\right)\right)-1=t\left(K_{2}\right) .
$$

This proof also works if the knots $K_{1}$ and $K_{2}$ are embedded in homology spheres (or any pair of compact 3-manifolds in which every closed embedded surface is separating). In general, however, it is important to keep in mind the delicacy of Theorem 3.21 (and Corollary 3.22), whose assumptions are encoded in Conventions 3.1, 3.3 and 3.9. In particular, the assumption of Convention 3.3 that $T$ and $\mathcal{G}$ are well-configured cannot always be satisfied as can be shown using straightforward examples in $S^{1} \times F$, where $F$ is a closed genus $g>1$ surface. Thus Corollary 3.22 cannot be applied to prove the following plausible conjecture in the case $g=1$ in any obvious way. 

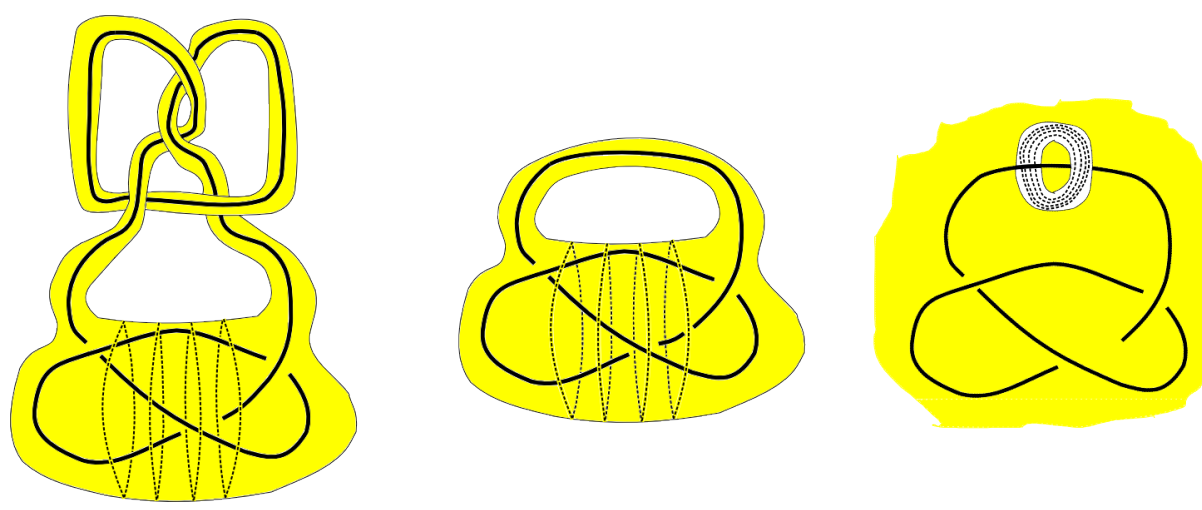

Figure 7: In the first diagram, $M_{2}$ (in yellow) is seen as situated in $E\left(K_{1} \#\right.$ $K_{2}, S^{3}$ ), and $\mathcal{G} \cap T$ is indicated with dashed lines lying on the "swallowfollow" torus $T$. In the second diagram, $M_{2}$ is re-embedded in $E\left(K_{2}, S^{3}\right)$, and in the final diagram, we see $M_{2}$ and $\mathcal{G} \cap T$ as they look after inverting the image of $T$ under this re-embedding.

Conjecture 4.2 Suppose $M$ is a compact 3-manifold and $T$ is a separating, incompressible, orientable, genus $g$ surface properly embedded in $M$. If $M_{1}$ and $M_{2}$ are the components of $E(T, M)$, then $g(M) \geq \max \left\{g\left(M_{1}\right), g\left(M_{2}\right)\right\}-g$.

Similarly, the need for $T$ and $\mathcal{G}$ to be well-configured is what keeps us from applying Theorem 3.21 and Corollary 3.22 to prove the analogue of Theorem 4.1 for satellite knots.

Theorem 4.1 has some relation to the "rank-genus conjecture" for knot complements in $S^{3}$. If we define $r(K)$ to be the minimal number of generators for $\pi_{1}\left(S^{3}-K\right)$, then the rank-genus conjecture states:

Conjecture 4.3 For all knots $K \subset S^{3}, r(K)=g\left(E\left(K, S^{3}\right)\right)=t(K)+1$.

Since a genus $g$ Heegaard splitting of a knot complement induces a $g$-generator presentation of $\pi_{1}\left(S^{3}-K\right)$, it is clear that $r(K) \leq t(K)+1$, but it remains unknown whether it is possible for this inequality to be strict. Boileau and Zieschang [1] described closed Seifert fibered 3-manifolds $M$ that satisfy $g(M)>r(M)$, where $r(M)$ is the rank of $\pi_{1}(M)$. More recently, Li [6] constructed closed hyperbolic 3-manifolds satisfying the same inequality.

Hence it seems likely that the rank-genus conjecture fails for knot complements, although it remains unknown. A pair of knots in $S^{3}$ whose tunnel number degenerated enough to violate Theorem 4.1 would have given a counterexample, since the following 
analogue of Theorem 4.1 for rank is trivial (thanks to Richard Weidmann for pointing out the simple line of proof below).

Proposition 4.4 For any knots $K_{1}, K_{2} \subset S^{3}, r\left(K_{1} \# K_{2}\right) \geq \max \left\{r\left(K_{1}\right), r\left(K_{2}\right)\right\}$.

Proof We have that $\pi_{1}\left(E\left(K_{1} \# K_{2}\right)\right)$ is an amalgamated free product $\pi_{1}\left(E\left(K_{1}\right)\right) * \mathbb{Z}$ $\pi_{1}\left(E\left(K_{2}\right)\right)$ that retracts onto each of its factors.

The fact that Theorem 4.1 is true indicates that the class of knot pairs that experience high tunnel number degeneration is not a good place to look for counterexamples to the rank-genus conjecture after all. In any event, the simplicity of the proof of Proposition 4.4 makes a striking contrast to our proof of Theorem 4.1.

Acknowledgments I would like to thank Maggy Tomova and Charlie Frohman for their support and guidance throughout my career. I would like to thank Jesse Johnson for the same, as well as for many helpful conversations and observations about this paper in particular.

\section{References}

[1] M Boileau, H Zieschang, Heegaard genus of closed orientable Seifert 3-manifolds, Invent. Math. 76 (1984) 455-468 MR746538

[2] F Bonahon, J-P Otal, Scindements de Heegaard des espaces lenticulaires, Ann. Sci. École Norm. Sup. 16 (1983) 451-466 MR740078

[3] T Kobayashi, A construction of arbitrarily high degeneration of tunnel numbers of knots under connected sum, J. Knot Theory Ramifications 3 (1994) 179-186 MR1279920

[4] T Kobayashi, Y Rieck, Heegaard genus of the connected sum of m-small knots, Comm. Anal. Geom. 14 (2006) 1037-1077 MR2287154

[5] T Kobayashi, Y Rieck, Knot exteriors with additive Heegaard genus and Morimoto's conjecture, Algebr. Geom. Topol. 8 (2008) 953-969 MR2443104

[6] T Li, Rank and genus of 3-manifolds, J. Amer. Math. Soc. 26 (2013) 777-829 MR3037787

[7] T Li, R Qiu, On the degeneration of tunnel numbers under a connected sum, Trans. Amer. Math. Soc. 368 (2016) 2793-2807

[8] Y Moriah, H Rubinstein, Heegaard structures of negatively curved 3-manifolds, Comm. Anal. Geom. 5 (1997) 375-412 MR1487722

[9] K Morimoto, There are knots whose tunnel numbers go down under connected sum, Proc. Amer. Math. Soc. 123 (1995) 3527-3532 MR1317043 
[10] K Morimoto, M Sakuma, Y Yokota, Examples of tunnel number one knots which have the property " $1+1=3$ ", Math. Proc. Cambridge Philos. Soc. 119 (1996) 113-118 MR1356163

[11] K Morimoto, J Schultens, Tunnel numbers of small knots do not go down under connected sum, Proc. Amer. Math. Soc. 128 (2000) 269-278 MR1641065

[12] J M Nogueira, Tunnel number degeneration under the connected sum of prime knots, Topology Appl. 160 (2013) 1017-1044 MR3049251

[13] F H Norwood, Every two-generator knot is prime, Proc. Amer. Math. Soc. 86 (1982) 143-147 MR663884

[14] T Saito, M Scharlemann, J Schultens, Lecture notes on generalized Heegaard splittings, preprint (2005) arXiv:math/0504167

[15] M Scharlemann, J Schultens, The tunnel number of the sum of $n$ knots is at least $n$, Topology 38 (1999) 265-270 MR1660345

[16] M Scharlemann, J Schultens, Annuli in generalized Heegaard splittings and degeneration of tunnel number, Math. Ann. 317 (2000) 783-820 MR1777119

[17] M Scharlemann, A Thompson, Thin position for 3-manifolds, from: "Geometric topology", (C Gordon, Y Moriah, B Wajnryb, editors), Contemp. Math. 164, Amer. Math. Soc. (1994) 231-238 MR1282766

[18] J Schultens, Additivity of tunnel number for small knots, Comment. Math. Helv. 75 (2000) 353-367 MR1793793

Department of Mathematics, Oklahoma State University

Stillwater, OK 74078, USA

trentschirmer@gmail.com

http://www.trentschirmer.com

Received: 3 December 2012 Revised: 3 August 2015 